\title{
INFERENCE ON THE QUANTILE REGRESSION PROCESS
}

\author{
ROGER KOENKER AND ZHIJIE XIAO \\ UNIVERSITY OF ILLINOIS AT URBANA-CHAMPAIGN
}

\begin{abstract}
Tests based on the quantile regression process can be formulated like the classical Kolmogorov-Smirnov and Crámer-von-Mises tests of goodness-of-fit employing the theory of Bessel processes as in ?. However, it is frequently desirable to formulate hypotheses involving unknown nuisance parameters, thereby jeopardizing the distribution free character of these tests. We characterize this situation as "the Durbin problem" since it was posed in ?, for parametric empirical processes.

In this paper we consider an approach to the Durbin problem involving a martingale transformation of the parametric empirical process suggested by ? and show that it can be adapted to a wide variety of inference problems involving the quantile regression process. In particular, we suggest new tests of the location shift and location-scale shift models that underlie much of classical econometric inference.

The methods are illustrated in some limited Monte-Carlo experiments and with a reanalysis of data on unemployment durations from the Pennsylvania Reemployment Bonus Experiments. The Pennsylvania experiments, conducted in 1988-89, were designed to test the efficacy of cash bonuses paid for early reemployment in shortening the duration of insured unemployment spells.
\end{abstract}

\section{INTRODUCTION}

Quantile regression is gradually evolving into a comprehensive approach to the statistical analysis of linear and nonlinear response models for conditional quantile functions. Just as classical linear regression methods based on minimizing sums of squared residuals enable one to estimate models for conditional mean functions $\Gamma$ quantile regression methods based on minimizing asymmetrically weighted absolute residuals offer a mechanism for estimating models for the conditional median function $\Gamma$ and the full range of other conditional quantile functions. By supplementing least squares estimation of conditional mean functions with techniques for estimating an entire family of conditional quantile functions $\Gamma$ quantile regression is capable of providing a much more complete statistical analysis of the stochastic relationships among random variables.

There is already a well-developed theory of asymptotic inference for many important aspects of quantile regression. Rank-based inference based on the approach of ? appears particularly attractive for a wide variety of quantile regression inference

Version: January 27, 2000. This version is preliminary and incomplete; please do not cite. The authors wish to express their appreciation to Jushan Bai whose work provided a vital initial impetus to this project. This research was partially supported by NSF grant SBR-9617206. 
problems including the construction of confidence intervals for individual quantile regression parameter estimates. There has also been considerable attention devoted to various resampling strategies. See e.g. ?Г? $\Gamma$ ? ?. In ? some initial steps have been taken toward a complete theory of inference based on the entire quantile regression process. These steps have clarified the close tie to classical Kolmogorov-Smirnov goodness of fit resultsTand related literature on Bessel processes initiated by ?. They have also successfully extended the applicability of certain Wald and rankscore tests to the linear location scale model.

This paper describes some further steps in this direction. These steps depend crucially on an ingenious suggestion by ? for dealing with tests of composite null hypotheses based on the empirical distribution function. Khmaladze's results have been slow to percolate into statistics generally lbut the approach has recently played an important role in work on regression diagnostics by ? and ?. In econometrics $\Gamma$ ? seems to have been the first to recognize the potential importance of these methods.

Khmaladze's martingale transformation approach provides a general strategy for purging the effect of estimated nuisance parameters from the first order asymptotic representation of the empirical process and thereby restoring the feasibility of "asymptotically distribution free" tests. The approach seems especially attractive in quantile regression settings and is capable of greatly expanding the scope of inference methods described in earlier work.

1.1. Quantile Treatment Effects. To motivate our results it is helpful to begin by reconsidering the classical two-sample treatment-control problem. In the simplest possible setting we can imagine a random sample of size $\Gamma n$ drawn from a homogeneous population and randomized into $n_{1}$ treatment observations $\Gamma$ and $n_{0}$ control observations. We have a response variable $\Gamma Y_{i} \Gamma$ and are interested in evaluating the effect of the treatment on this reponse.

In a typical clinical trial application Ifor exampleIthe treatment would be some form of medical procedureTand $Y_{i}$ Tmight be $\log$ survival time. In our application appearing in Section 6 The treatment is an offer of a cash bonus for early exit from a spell of unemployment $\Gamma$ and $Y_{i}$ is the logarithm of individual $i$ 's unemployment duration. In the first instance we might be satisfied to know simply the mean treatment effect Tthat is $\Gamma$ the difference in means for the two groups. This we could evaluate by "running the regression" of the observed $y_{i}$ 's on an indicator variable: $x_{i}=1$ Tif subject $i$ was treated $\Gamma x_{i}=0 \Gamma$ if subject $i$ was a control. Of course this regression would presume $\Gamma$ implicitly that the variability of the two subsamples was the same; this observation opens door to the possibility that the treatment alters other features of the response distribution as well. Although we are accustomed to thinking about regression models in which the covariates affect only the location of the conditional distribution of the response - this is force of the iid error assumption - there is no compelling reason to believe that covariates must operate in this restrictive fashion.

? introduced the following general formulation of the two sample treatment effect $\Gamma$ 
"Suppose the treatment adds the amount $\Delta(x)$ when the response of the untreated subject would be $x$. Then the distribution $G$ of the treatment responses is that of the random variable $X+\Delta(X)$ where $X$ is distributed according to $F . "$

? provides a detailed axiomatic analysis of this formulation $\Gamma$ showing that if we define $\Delta(x)$ as the "horizontal distance" between $F$ and $G$ at $x \Gamma$ so

$$
F(x)=G(x+\Delta(x))
$$

then $\Delta(x)$ is uniquely defined and can be expressed as

$$
\Delta(x)=G^{-1}(F(x))-x .
$$

Changing variablesTso $\tau=F(x)$ we obtain what we will call the quantile treatment effect $\Gamma$

$$
\delta(\tau)=\Delta\left(F^{-1}(\tau)\right)=G^{-1}(\tau)-F^{-1}(\tau)
$$

In the two sample setting this quantity is naturally estimable by

$$
\hat{\delta}(\tau)=\hat{G}_{n_{1}}^{-1}(\tau)-\hat{F}_{n_{0}}^{-1}(\tau)
$$

where $\hat{G}_{n_{1}}, \hat{F}_{n_{0}}$ denote the empirical distribution functions of the treatment and control observations respectivelyTand $\hat{F}_{n}^{-1}=\inf \left\{x \mid \hat{F}_{n}(x) \geq \tau\right\}$ Tas usual. Since we cannot observe subjects in both the treated and control states - and this platitude may be regarded as the fundamental "uncertainty principle" underlying the the "causal effects" literature - it seems reasonable to regard $\delta(\tau)$ as a complete description of the treatment effect. Of course $\mathrm{Tthere}$ is no way of really knowing whether the treatment operates in the way prescribed by Lehmann. In fact $\Gamma$ the treatment may make otherwise weak subjects especially robustTand turn the strong to jello. All we can observe from the experimental evidence is the difference between the two marginal survival distributions $\Gamma$ so it is natural to associate the treatment effect with this difference. The quantile treatment effect provides the unexpurgated version.

Of course $\Gamma$ it is possible that the two distributions differ only by a location shift $\Gamma$ so $\delta(\tau)=\delta_{0}$ Tor that they differ by a scale shift so $\delta(\tau)=\delta_{1} F^{-1}(\tau)$ or that they differ by a location and scale shift so $\delta(\tau)=\delta_{0}+\delta_{1} F^{-1}(\tau)$. But these hypotheses are all nicely nested within Lehmann's general framework. And yet $\Gamma$ as we shall see $\Gamma$ testing these hypotheses against the general alternatives represented by the Lehmann- Doksum quantile treatment effect poses some serious techical problems.

In the next section we briefly describe the nature of these problems in their canonical form Tthe classical one-sample goodness of fit problem. Khmaladze's martingale decomposition strategy for dealing with these problems is then introduced. Section 3 extends the Khmaladze approach to general problems of inference based on the quantile regression process. Section 4 treats some practical problems of implementing the tests. Section 5 reports the results of a limited Monte-Carlo experiment designed to evaluate the finite sample performance of the tests. Section 6 describes an empirical 
application to the analysis of unemployment durations. And Section 7 contains some concluding remarks.

\section{A Heuristic Introduction to Khmaladzation}

Arguably the most fundamental problem of statistical inference is the classical goodness-of-fit problem: given a random sample $\Gamma\left\{y_{1}, \ldots, y_{n}\right\}$ Ton a real-valued random variable $\Gamma Y$ Test the hypothesis that $Y$ comes from distribution function $\Gamma F_{0}$. Tests based on the empirical distribution function $\Gamma F_{n}(y)=n^{-1} \sum I\left(Y_{i} \leq y\right)$ Гlike the Kolmogorov-Smirnov statistic

$$
K_{n}=\sup _{x \in \mathbb{R}} \sqrt{n}\left|F_{n}(y)-F_{0}(y)\right|,
$$

are especially attractive because they are asymptotically distribution-free. The limiting distribution of $K_{n}$ is the same for every continuous distribution function $F_{0}$. This remarkable fact follows by (trivially) noting that the process $\Gamma \sqrt{n}\left(F_{n}(y)-F_{0}(y)\right) \Gamma$ can be transformed to a test of uniformity $\mathrm{Tvia}$ the change of variable $\Gamma y \rightarrow F_{0}^{-1}(t) \Gamma$ based on

$$
v_{n}(t)=\sqrt{n}\left(F_{n}\left(F_{0}^{-1}(t)\right)-t\right) .
$$

It is well known that $v_{n}(t)$ converges weakly to a Brownian bridge process $\Gamma v_{0}(t) \Gamma$ that is a mean-zero Gaussian process with covariance function

$$
E v_{0}(t) v_{0}(s)=t \wedge s-s t
$$

and thus the distribution of $K_{n}$ and related functionals follows from the observation of $?$ and its subsequent refinements.

2.1. The Durbin Problem. It is rare in practice Thowever $\Gamma$ that we are willing to specify $F_{0}$ completely. More commonly family $\mathcal{F}_{\theta}$ with $\theta \in \Theta \subseteq \mathbb{R}^{p}$. For example $\Gamma$ we may wish to test "normality" $\Gamma$ claiming that $Y$ has distribution $F_{\theta_{0}}(y)=\Phi\left(\left(y-\mu_{0}\right) / \sigma_{0}\right) \Gamma$ but $\theta_{0}=\left(\mu_{0}, \sigma_{0}\right)$ is unknown. We are thus led to consider $\Gamma$ following ? Tthe parametric empirical process $\Gamma$

$$
U_{n}(y)=\sqrt{n}\left(F_{n}(y)-F_{\hat{\theta}_{n}}(y)\right) .
$$

Again changing variables $\Gamma$ so $y \rightarrow F_{\theta_{0}}^{-1}(t)$, we may equivalently consider

$$
u_{n}(t)=\sqrt{n}\left(G_{n}(t)-G_{\hat{\theta}_{n}}(t)\right)
$$

where $G_{n}(t)=F_{n}\left(F_{\theta_{0}}^{-1}(t)\right)$ and $G_{\hat{\theta}}(t)=F_{\hat{\theta}_{n}}\left(F_{\theta_{0}}^{-1}(t)\right)$ so $G_{\theta_{0}}(t)=t$. Under mild conditions on the sequence $\left\{\hat{\theta}_{n}\right\}$ we have the linear (Bahadur) representation $\Gamma$

$$
\sqrt{n}\left(\hat{\theta}_{n}-\theta_{0}\right)=\int_{0}^{1} h_{0}(s) d v_{n}(s)+o_{p}(1) .
$$


So provided the mapping $\theta \rightarrow G_{\theta}$ has a Fréchet derivative ${ }^{1} \Gamma g=g_{\theta_{0}} \Gamma$ we may write

$$
G_{\hat{\theta}_{n}}(t)=t+\left(\hat{\theta}_{n}-\theta_{0}\right)^{\top} g(t)+o_{p}(1),
$$

and thus obtain with $r_{n}(t)=o_{p}(1)$,

$$
\begin{aligned}
\hat{v}_{n}(t) & =\sqrt{n}\left(G_{n}(t)-t-\left(G_{\hat{\theta}_{n}}(t)-t\right)\right) \\
& =v_{n}(t)-g(t)^{\top} \int_{0}^{1} h_{0}(s) d v_{n}(s)+r_{n}(t),
\end{aligned}
$$

which converges weakly to the Gaussian process $\Gamma$

$$
u_{0}(t)=v_{0}(t)-g(t)^{\top} \int_{0}^{1} h_{0}(s) d v_{0}(s) .
$$

The necessity of estimating $\theta_{0}$ introduces the drift component $g(t)^{\top} \int_{0}^{1} h_{0}(s) d v_{0}(s)$. Instead of the simple Brownian bridge process $\Gamma v_{0}(t) \Gamma$ we obtain a more complicated Gaussian process with covariance function

$$
E u_{0}(t) u_{0}(s)=s \wedge t-t s-g(t)^{\top} \mathcal{H}_{0}(s)-g(s)^{\top} \mathcal{H}_{0}(t)+g(s)^{\top} \mathcal{J}_{0} g(t)
$$

where $\mathcal{H}_{0}(t)=\int_{0}^{t} h_{0}(s) d s$ and $\mathcal{J}_{0}=\int_{0}^{1} \int_{0}^{1} h_{0}(t) h_{0}(s)^{\top} d t d s$. When $\hat{\theta}_{n}$ is the mleTso $h_{0}(s)=-\left(E \nabla_{\theta} \psi\right)^{-1} \psi\left(F^{-1}(s)\right)$ with $\psi=\nabla_{\theta} \log f$ The covariance function simplifies nicely to

$$
E u_{0}(t) u_{0}(s)=s \wedge t-t s-g(s)^{\top} \mathcal{I}_{0} g(t)
$$

where $\mathcal{I}_{0}$ denotes Fisher's information matrix. See ? and ? for further details on this case.

The practical consequence of the drift term involving the function $g(t)$ is to invalidate the distribution-free character of the original test. Tests based on the parametric empirical process $u_{n}(t)$ require special consideration of the process $u_{0}(t)$ and its dependence on $F$ in each particular case. ? discuss several leading examples. ? describes a general numerical approach based on Fourier inversion $\Gamma$ but also expresses doubts about feasibility of the method when the parametric dimension $\Gamma p$, of $\theta$ exceeds one. Although the problem of finding a viable ggeneral approach to inference based on the parametric empirical process had been addressed by several previous authors Tnotably ? Twe will in the spirit of Stigler's law of eponymyГ? Trefer to this as "the Durbin problem."

2.2. Martingales and the Doob-Meyer Decomposition. Khmaladze's general approach to the Durbin problem can be motivated as a natural elaboration of the Doob-Meyer decomposition for the parametric empirical process. Recall that a stochastic process $x=\{x(t): t \geq 0\}$ that is (i) right continuous with left limits; (ii)

\footnotetext{
${ }^{1}$ That is, $\sup _{t}\left|G_{\theta+h}(t)-G_{\theta}(t)-h^{\top} g(t)\right|=o(\|h\|)$ as $h \rightarrow 0$, see van der Vaart (1998, p 278.)
} 
integrable $\sup _{0 \leq t<\infty} E|x(t)|<\infty$; and (iii) adapted to the filtration $\left\{\mathcal{F}_{t}: t \geq 0\right\} \Gamma$ is called a submartingale if

$$
E\left(x(t+s) \mid \mathcal{F}_{t}\right) \geq x(t) \quad \text { a.s. }
$$

and is called a martingale if

$$
E\left(x(t+s) \mid \mathcal{F}_{t}\right)=x(t) \quad \text { a.s. }
$$

The Doob-Meyer decomposition asserts that for any nonnegative submartingale $\Gamma x \Gamma$ there exists an increasing right continuous predictable process $\Gamma a(t) \Gamma$ such that $E a(t)<$ $\infty$ Tand a right continuous martingale $m$, such that

$$
x(t)=m(t)+a(t) \quad a . s .
$$

A process $a(t)$ is called predictable with respect to a filtration $\left\{\mathcal{F}_{t}: t \geq 0\right\}$ if $\Gamma$ viewed as a mapping from $[0, \infty) \times \Omega$ to $\mathbb{R}$ it is measurable with respect to the $\sigma$-algebra generated by the filtration $\mathcal{F}_{t}$ That is the $\sigma$-algebra generated by all sets of the form $(r, s] \times A$ for $0 \leq a<b<1$ and $A \in \mathcal{F}_{r}$. See e.g. ?.

Let $X_{1}, \ldots, \bar{X}_{n}$ be iid from $F_{0} \Gamma$ so $Y_{i}=F_{0}\left(X_{i}\right), \quad i=1, \ldots, n$ are iid uniform $\Gamma$ $U[0,1]$. The empirical distribution function

$$
G_{n}(t)=F_{n}\left(F_{0}^{-1}(t)\right)=n^{-1} \sum_{i=1}^{n} I\left(Y_{i} \leq t\right) .
$$

viewed as a process $\Gamma$ is a submartingale. We have an associated filtration $\mathcal{F}^{G_{n}}=$ $\left\{\mathcal{F}_{t}^{G_{n}}: 0 \leq t \leq 1\right\}$ and the order statistics $Y_{(1)}, \ldots, Y_{(n)}$ are Markov times with respect to $\mathcal{F}^{G_{n}}$ Tthat is $\left\{X_{(i)} \leq t\right\}=\left\{F_{n}(t) \geq i / n\right\} \in \mathcal{F}_{t}^{G_{n}}$.

The process $G_{n}(t)$ is Markov; Khmaladze notes that for $\Delta t \geq 0 \Gamma$

$$
\begin{aligned}
n \Delta G_{n}(t) & =n\left[G_{n}(t+\Delta t)-G_{n}(t)\right] \\
& \sim \operatorname{Binomial}\left(n\left(1-G_{n}(t)\right), \Delta t /(1-t)\right)
\end{aligned}
$$

with $G_{n}(0)=0$ Tthus

$$
E\left(\Delta G_{n}(t) \mid \mathcal{F}_{t}^{G_{n}}\right)=\frac{1-G_{n}(t)}{1-t} \Delta t
$$

This suggests the decomposition

$$
G_{n}(t)=\int_{0}^{t} \frac{1-G_{n}(s)}{1-s} d s+m_{n}(t) .
$$

That $m_{n}(t)$ is a martingale then follows from the fact that $\Gamma$ from $(2.2) \Gamma$

$$
E\left(m_{n}(t) \mid \mathcal{F}_{s}^{G_{n}}\right)=m_{n}(s)
$$

and integrability of $m_{n}(t)$ follows from the inequality

$$
\int_{0}^{t} \frac{1-G_{n}(s)}{1-s} d s \leq-\log \left(1-Y_{(n)}\right),
$$


which implies a finite mean for the compensator $\Gamma$ or predictable component. Substituting for $G_{n}(t)$ in (2.2) we have the classical Doob-Meyer decomposition of the empirical process $v_{n}$

$$
v_{n}(t)=w_{n}(t)-\int_{0}^{t} \frac{v_{n}(s)}{1-s} d s
$$

where $v_{n}(t)=\sqrt{n}\left(G_{n}(t)-t\right)$ and the normalized process $w_{n}(t)=\sqrt{n} m_{n}(t)$ converges weakly to a standard Brownian motion process $\Gamma w_{0}(t)$, by the argument of Khmaladze(1981Г $\S 2.6)$.

2.3. The Parametric Empirical Process. To extend this approach to the general parametric empirical process $\Gamma$ we now let $g(t)=\left(t, \bar{g}(t)^{\top}\right)^{\top}=\left(t, g_{1}(t), \ldots g_{m}(t)\right)^{\top}$ be a $(m+1)$-vector of real-valued functions on [0,1]. Suppose that the functions $\dot{g}(t)=d g(t) / d t$ are linearly independent in a neighborhood of 1 so

$$
C(t) \equiv \int_{t}^{1} \dot{g}(s) \dot{g}(s)^{\top} d s
$$

is non-singularTand consider the transformation

$$
w_{n}(t)=v_{n}(t)-\int_{0}^{t} \dot{g}(s)^{\top} C^{-1}(s) \int_{s}^{1} \dot{g}(r) d v_{n}(r) d s .
$$

Here $\Gamma w_{n}(t)$ clearly depends upon the choice of $g \Gamma$ and therefore differs from $w_{n}(t)$ defined above. But the abuse of notation maybe justified by noting that in the special case $g(t)=t$ Wwe have $C(s)=1-s$ Tand $\int_{s}^{1} \dot{g} d v_{n}(r)=-v_{n}(s)$ yielding the Doob-Meyer decomposition (2.2). In the general case $\Gamma$ the transformation

$$
Q_{g} \varphi(t)=\varphi(t)-\int_{0}^{t} \dot{g}(s)^{\top} C^{-1}(s) \int_{s}^{1} \dot{g}(r) d \varphi(r) d s
$$

may be recognized as the residual from the prediction of $\varphi(t)$ based on the recursive least squares estimate using information from $(t, 1]$. For functions in the span of $g \Gamma$ the prediction is exact $\Gamma$ that is $\Gamma Q_{g} g=0$.

Now returning to the representation of the parametric empirical process $\Gamma \hat{v}_{n}(t) \Gamma$

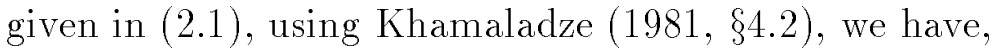

$$
\begin{aligned}
\tilde{v}_{n}(t) & =Q_{g} \hat{v}_{n}(t) \\
& =Q_{g}\left(v_{n}(t)-\bar{g}(t)^{\top} \int_{0}^{1} h_{0}(s) d v_{n}(s)+r_{n}(t)\right) \\
& =Q_{g}\left(v_{n}(t)+r_{n}(t)\right) \\
& =w_{0}(t)+o_{p}(1) .
\end{aligned}
$$

The transformation of the parametric empirical process annihilates the $g$ component of the representation and in so doing restores the feasibility of asymptotically distribution free tests based on the transformed process $\tilde{v}_{n}(t)$. 
2.4. The Parametric Empirical Quantile Process. What can be done for tests based on the parametric empirical process can also be adapted for tests based on the parametric empirical quantile process. In some ways the quantile domain is actually more convenient. Suppose $\left\{y_{1}, \ldots, y_{n}\right\}$ constitute a random sample on $Y$ with distribution function $F_{Y}$. Consider testing the hypothesis $\Gamma F_{Y}(y)=F_{0}\left(\left(y-\mu_{0}\right) / \sigma_{0}\right)$, so $\Gamma$

$$
\alpha(t) \equiv F_{Y}^{-1}(t)=\mu_{0}+\sigma_{0} F_{0}^{-1}(t) .
$$

Given the empirical quantile process

$$
\hat{\alpha}(t)=\inf \left\{a \in \mathbb{R} \mid \sum_{i=1}^{n} \rho_{\tau}\left(y_{i}-a\right)=\min !\right\}
$$

and known parameters $\theta_{0}=\left(\mu_{0}, \sigma_{0}\right)$ tests may be based on

$$
v_{n}(t)=\sqrt{n}(\hat{\alpha}(t)-\alpha(t)) / \sigma s_{0}(t) \Rightarrow v_{0}(t)
$$

where $s_{0}(t)=\left(f_{0}\left(F_{0}^{-1}(\tau)\right)\right)^{-1}$ and $v_{0}(\tau)$ is the Brownian bridge process.

To test our hypothesis when $\theta$ is unknown $\Gamma$ set $\xi(t)=\left(1, F_{0}^{-1}(t)\right)^{\top}$ and for an estimator $\hat{\theta}_{n}$ satisfying $\Gamma$

$$
\sqrt{n}\left(\hat{\theta}_{n}-\theta_{0}\right)=\int_{0}^{1} h_{0}(s) d v_{n}(s)+o_{p}(1)
$$

set

$$
\tilde{\alpha}(t)=\hat{\mu}+\hat{\sigma} F_{0}^{-1}(t)=\hat{\theta}_{n}^{\top} \xi(t)
$$

Then

$$
\begin{aligned}
\hat{v}_{n}(t) & =\sqrt{n}(\hat{\alpha}(t)-\tilde{\alpha}(t) /(\sigma s(t)) \\
& =\sqrt{n}(\hat{\alpha}(t)-\alpha(t)-(\hat{\alpha}(t)-\alpha(t))) /(\sigma s(t)) \\
& =v_{n}(t)-\sqrt{n}\left(\hat{\theta}-\theta_{0}\right)^{\top} \xi(t) /(\sigma s(t)) \\
& =v_{n}(t)-\xi(t)^{\top} \int_{0}^{1} h_{0}(s) d v_{n}(s)+o_{p}(1)
\end{aligned}
$$

Thus Tif we take $g(t)=\left(t, \xi(t)^{\top} / s(t)\right)^{\top}$ Twe obtain $\Gamma$

$$
\dot{g}(t)=\left(1, \dot{f} / f, 1+F_{0}^{-1}(t) \dot{f} / f\right)^{\top}
$$

where $\dot{f} / f$ is evaluated at $F_{0}^{-1}(t)$ so for example in the Gaussian case $\Gamma$

$$
\dot{g}(t)=\left(1-\Phi^{-1}(t), 1-\Phi^{-1}(t)^{2}\right)^{\top} .
$$

Given the representation (2.3) and the fact that $\xi(t)$ lies in the linear span of $g \Gamma$ we may again apply Khmaladze's martingale transformation to obtain $\Gamma$

$$
\tilde{v}_{n}(t)=Q_{g} \hat{v}_{n}(t)
$$


which can then be shown to converge to the standard Brownian motion process. In the next section we explore extending this approach to multidimensional quantile regression.

\section{Quantile Regression InfEREnCE}

The classical linear regression model asserts that the conditional mean of the response $\Gamma Y_{i}$ Tgiven covariates $\Gamma x_{i} \Gamma$ may be expressed as a linear function of the covariates. That is $\Gamma$ there exists a $\beta \in \mathbb{R}^{P}$ such that $\Gamma$

$$
E\left(y_{i} \mid x_{i}\right)=x_{i}^{\top} \beta .
$$

The linear quantile regression model asserts Tanalogously that the conditional quantile functions of $y_{i}$ given $x_{i}$ are linear in covariates $\Gamma$

$$
F_{y_{i} \mid x_{i}}^{-1}\left(\tau \mid x_{i}\right)=x_{i}^{\top} \beta(\tau)
$$

for $\tau$ in some index set $\mathcal{T} \subset[0,1]$. The model (3.1) will be taken to be our basic maintained hypothesis. For convenience we will restrict attention to the case that $\mathcal{T}=[\epsilon, 1-\epsilon]$ for some $\epsilon \in(0,1 / 2)$ Tand to faciliatate asymptotic local power analysis we will consider sequences of models for which $\beta(\tau)=\beta_{n}(\tau)$ depends explicitly on the sample size $\Gamma n$.

A leading special case is the location-scale shift model $\Gamma$

$$
F_{y_{i} \mid x_{i}}^{-1}\left(\tau \mid x_{i}\right)=x_{i}^{\top} \alpha+x_{i}^{\top} \gamma F_{0}^{-1}(\tau) \text {. }
$$

where $F_{0}^{-1}(\tau)$ denotes a univariate quantile function. Covariates affect both the location and scale of the conditional distribution of $y_{i}$ given $x_{i}$ in this model covariates have no effect on the shape of the conditional distribution. Typically vectors $\left\{x_{i}\right\}$ "contain an intercept" so e.g. $\Gamma x_{i}=\left(1, z_{i}^{\top}\right)^{\top}$ and $(3.2)$ may be seen as arising from the linear model

$$
y_{i}=x_{i}^{\top} \alpha+\left(x_{i}^{\top} \gamma\right) u_{i}
$$

where the "errors" $\left\{u_{i}\right\}$ are iid with distribution function $F_{0}$. Further specializing the model Tmay write $\Gamma$

$$
x_{i}^{\top} \gamma=\gamma_{0}+z_{i}^{\top} \gamma_{1}
$$

and the restriction $\Gamma \gamma_{1}=0$ Then implies that the covariates affect only the location of the $y_{i}$ 's. We will call this model

$$
F_{y_{i} \mid x_{i}}^{-1}\left(\tau \mid x_{i}\right)=x_{i}^{\top} \alpha+\gamma_{0} F_{0}^{-1}(\tau)
$$

the location shift model. Although this model underlies much of classical econometric inference $\mathrm{Tit}$ posits a very narrowly circumscribed role for the $x_{i}$. In the remainder of this section we explore ways to test the hypotheses that the general linear quantile regression model takes either the location shift or location-scale shift form. 
We will consider a linear hypothesis of the general form $\Gamma$

$$
R \beta(\tau)-r=\Psi(\tau) \quad \tau \in \mathcal{T}
$$

where $R$ denotes a $q \times p$ matrix $\Gamma q \leq p, r \in \mathbb{R}^{q} \Gamma$ and $\Psi(\tau)$ denotes a known function $\Psi: \mathcal{T} \rightarrow \mathbb{R}^{q}$. For example in the one sample setting of the previous section $\Gamma$ we might take $R=\sigma^{-1}, r=\mu / \sigma$ and $\Psi(\tau)=\Phi^{-1}(\tau)$, in order to test that the $y_{i}$ 's were $\mathcal{N}\left(\mu, \sigma^{2}\right)$.

In the two sample model described in Section 1.1.

$$
F_{y_{i} \mid D_{i}}^{-1}\left(\tau \mid D_{i}\right)=\beta_{0}(\tau)+\beta_{1}(\tau) D_{i}
$$

we might like to test that The treatment and control distributions differ by an affine transformation

$$
\beta_{0}(\tau)=\theta_{0}+\theta_{1} \beta_{1}(\tau)
$$

orTeven more simply that they differ by a location shift $\Gamma$

$$
\beta_{0}(\tau)=\theta_{0}+\beta_{1}(\tau)
$$

In these cases we may take $\Psi(\tau) \equiv 0, r=\theta_{0}, R=\left(1,-\theta_{1}\right)$ in the former case $\Gamma$ and $R=(1,-1)$ in the latter case. Of course $\Gamma$ we could also expand these two-sample hypotheses to consider fully specified parametric models with an explicit choice of $\Psi(\tau)$ Thowever $\Gamma$ the semi-parametric form of the hypotheses expressed above seems more plausible for most econometric applications.

We will consider tests based on the quantile regression process $\Gamma$

$$
\hat{\beta}(\tau)=\operatorname{argmin}_{b \in \mathbb{R}^{p}} \sum_{i=1}^{n} \rho_{\tau}\left(y_{i}-x_{i}^{\top} b\right)
$$

where $\rho_{\tau}(u)=u(\tau-I(u<0))$. Under the location-scale shift form of the quantile regression model (3.2) we will have under mild regularity conditions $\Gamma$

$$
\sqrt{n} f_{0}\left(F_{0}^{-1}(\tau)\right) \Omega^{-1 / 2}(\hat{\beta}(\tau)-\beta(\tau)) \Rightarrow v_{0}(\tau)
$$

where $v_{0}(\tau)$ now denotes a $p$-dimensional independent Brownian bridge process $\Gamma$

$$
\beta(\tau)=\alpha+\gamma F^{-1}(\tau),
$$

and $\Omega=H_{0}^{-1} J_{0} H_{0}^{-1}$ with $J_{0}=\lim n^{-1} \sum x_{i} x_{i}^{\top}$, and $H_{0}=\lim n^{-1} \sum x_{i} x_{i}^{\top} / \gamma^{\top} x_{i}$.

It then follows quite easily that under the null hypothesis (3.4) $\Gamma$

$$
v_{n}(\tau)=\sqrt{n} f_{0}\left(F_{0}^{-1}(\tau)\right)\left(R \Omega R^{\top}\right)^{-1 / 2}(R \hat{\beta}(\tau)-r-\Psi(\tau)) \Rightarrow v_{0}(\tau) .
$$

So tests that were asymptotically distribution free could be readily constructed. Indeed $\Gamma$ ? consider tests of this type when $R$ constitutes an exclusion restriction so e.g. $\Gamma R=\left[0: I_{q}\right], r=0 \Gamma$ and $\Psi(\tau)=0$. In such cases it is also shown that the nuisance parameters $f_{0}\left(F_{0}^{-1}(\tau)\right)$ and $\Omega$ can be replaced by consistent estimates without jeopardizing the distribution free character of the tests. 
To formalize the foregoing discussion we introduce the following conditions Twhich closely resemble the conditions employed in Koenker and Machado. We will assume that the $\left\{y_{i}\right\}$ 's are conditional on $x_{i}$ Tindependent with linear conditional quantile functions given by (3.1) and localTin a sense specified in A.3T to the location-scale shift model (3.2).

A. 1. The distribution function $F_{0}$, in (3.2) has a continuous Lesbesgue density, $f_{0}$, with $f_{0}(u)>0$ on $\left\{u: 0<F_{0}(u)<1\right\}$.

A. 2. The sequence of design matrices $\left\{X_{n}\right\}=\left\{\left(x_{i}\right)_{i=1}^{n}\right\}$ satisfy:

(i): $x_{i 1} \equiv 1 \quad i=1,2, \ldots$

(ii): $J_{n}=n^{-1} X_{n} X_{n} \rightarrow J_{0}$, a positive definite matrix.

(iii): $H_{n}=n^{-1} X_{n} \Gamma_{n}^{-1} X_{n} \rightarrow H_{0}$, a positive definite matrix where $\Gamma_{n}=\operatorname{diag}\left(\gamma^{\top} x_{i}\right)$. (iv): $\max _{i=1, \ldots, n}\left\|x_{i}\right\|=\mathcal{O}\left(n^{1 / 4} \log n\right)$

A. 3. There exists a fixed, continuous function $\zeta(\tau):[0,1] \rightarrow \mathbb{R}^{q}$ such that for samples of size $n$,

$$
R \beta_{n}(\tau)-r-\Psi(\tau)=\zeta(\tau) / \sqrt{n}
$$

As noted in ? Tconditions A.1 and A.2 are quite standard in the quantile regression literature. Somewhat weaker conditions are employed by ? in an effort to extend the theory further into the tails. But this isn't required for our present purposes $\Gamma$ so we have reverted to conditions closer to those of ?. Condition A.3 enables us to explore local asymptotic power of the proposed tests employing a rather general form for the local alternatives.

We can now state our first result. Proofs of all results appear in the appendix.

Theorem 1. Let $\mathcal{T}$ denote the closed interval $[\varepsilon, 1-\varepsilon]$, for some $\varepsilon \in(0,1 / 2)$. Under conditions A.1-3

$$
v_{n}(\tau) \Rightarrow v_{0}(\tau)+\eta(\tau) \text { for } \tau \in \mathcal{T}
$$

where $v_{0}(\tau)$ denotes a $q$-variate standard Brownian bridge process and

$$
\eta(\tau)=f_{0}\left(F_{0}^{-1}(\tau)\right)\left(R \Omega R^{\top}\right)^{-1 / 2} \zeta(\tau) .
$$

Under the null hypothesis, $\zeta(\tau)=0$, the test statistic

$$
\sup _{\tau \in \mathcal{T}}\left\|v_{n}(\tau)\right\| \Rightarrow \sup _{\tau \in \mathcal{T}}\left\|v_{0}(\tau)\right\| \text {. }
$$

TypicallyTeven if the hypothesis is fully specified $\Gamma i t$ is necessary to estimate the matrix $\Omega$ and the function $\varphi(t) \equiv f_{0}\left(F_{0}^{-1}(t)\right)$. Fortunately replaced by estimates satisfying the following condition.

A. 4. There exist estimators $\varphi_{n}(\tau)$ and $\Omega_{n}$ satisfying

i.: $\sup _{\tau \in \mathcal{T}}\left|\varphi_{n}(\tau)-\varphi_{0}(\tau)\right|=o_{p}(1)$,

ii.: $\left\|\Omega_{n}-\Omega\right\|=o_{p}(1)$.

[Recall that we need to define matrix norms a la Hilbert-Schmidt.] 
Corollary 1. The conclusions of Theorem 1 remain valid if $f_{0}\left(F_{0}^{-1}(\tau)\right)$ and $\Omega$ are replaced by estimates satisfying condition $A .4$.

Theorem 1 extends slightly the results of ? Tbut it fails to answer our main question: how to deal with unknown nuisance parameters in $R$ and $r$ ? To begin to address this question we introduce the following condition.

A. 5. There exist estimators $R_{n}$ and $r_{n}$ satisfying $\sqrt{n}\left(R_{n}-R\right)=\mathcal{O}_{p}(1)$ and $\sqrt{n}\left(r_{n}-\right.$ $r)=\mathcal{O}_{p}(1)$.

And we consider the parametric quantile regression process $\Gamma$

$$
\hat{v}_{n}(\tau)=\sqrt{n} f_{0}\left(F_{0}^{-1}(\tau)\right)\left[R_{n} \Omega R_{n}^{\top}\right]^{-1 / 2}\left(R_{n} \hat{\beta}(\tau)-r_{n}-\Psi(\tau)\right) .
$$

The next result establishes a representation for $\hat{v}_{n}(\tau)$ analogous that provided in $(2.2)$ for the univariate empirical quantile process.

Theorem 2. Under conditions A.1-5, we have

$$
\hat{v}_{n}(\tau)-\xi(\tau)^{\top} Z_{n} \Rightarrow v_{0}(\tau)+\eta(\tau)
$$

where $\xi(\tau)=f_{0}\left(F_{0}^{-1}(\tau)\right)\left(1, F_{0}^{-1}(\tau)\right)^{\top}$, and $Z_{n}=\mathcal{O}_{p}(1)$, with $v_{0}(\tau)$ and $\eta(\tau)$ as specified in Theorem 1.

Corollary 2. The conclusions of Theorem 2 remain valid if $f_{0}\left(F_{0}^{-1}(\tau)\right)$ and $\Omega$ are replaced by estimates satisfying condition $A .4$.

As in the univariate case we are faced with two options. We can accept the presence of the $Z_{n}$ term Tand abandon the asymptotically distribution free nature of tests based upon $\hat{v}_{n}(\tau)$. Or we can $\Gamma$ following KhmaladzeTtry to find a transformation of $\hat{v}_{n}(\tau)$ that annihilates the $Z_{n}$ contribution Tand thus restores the asymptotically distribution free nature of inference. We adopt the latter approach.

Let $g(t)=\left(t, \xi(t)^{\top}\right)^{\top}$ so

$$
\dot{g}(t)=\left(1, \psi(t), \psi(t) F^{-1}(t)\right)^{\top}
$$

with $\psi(t)=\dot{f} / f\left(F^{-1}(t)\right)$. We will assume that $g(t)$ satisfies the following condition.

A. 6. The function $g(t)$ satisfies:

i: $\int\|\dot{g}(t)\|^{2} d t<\infty$,

ii: $\left\{\dot{g}_{i}(t): i=1, \ldots, m\right\}$ are linearly independent in a neighborhood of 1 .

We consider the transformed process $\Gamma$

$$
\tilde{v}_{n}(\tau) \equiv Q_{g} \hat{v}_{n}(\tau)=\hat{v}_{n}(\tau)-\int_{0}^{\tau} \dot{g}(s) C^{-1}(s) \int_{s}^{1} \dot{g}(r) d \hat{v}_{n}(r) d s,
$$

where the recursive least squares transformation should now be interpreted as operating coordinate by coordinate on the $\hat{v}_{n}$ process. 
Theorem 3. Under conditions A.1 - 6, we have

$$
\tilde{v}_{n}(\tau) \Rightarrow w_{0}(\tau)+\tilde{\eta}(\tau)
$$

where $w_{0}(\tau)$ denotes a q-variate standard Brownian motion, and $\tilde{\eta}(\tau)=Q_{g} \eta(\tau)$. Under the null hypothesis, $\zeta(\tau)=0$,

$$
\sup _{\tau \in \mathcal{T}}\left\|\tilde{v}_{n}(\tau)\right\| \Rightarrow \sup _{\tau \in \mathcal{T}}\left\|w_{0}(\tau)\right\| \text {. }
$$

[Note: Khmaladze $(1981, \S 3.3)$ shows that A.6.ii implies $C^{-1}(\tau)$ exists for all $\left.\tau.\right]$

Typically in applications $\Gamma$ the function $g(t)$ will not be specified under the null hypothesis $\Gamma$ but will also need to be estimated. Fortunately $\Gamma$ only one rather mild further condition is needed to enable us to replace $g$ by an estimate.

A. 7. There exists an estimator, $g_{n}(\tau)$, satisfying

$$
\sup _{\tau \in \mathcal{T}}\left\|\dot{g}_{n}(\tau)-\dot{g}(\tau)\right\|=o_{p}(1) .
$$

Corollary 3. The conclusions of Theorem 3 remain valid if $f\left(F^{-1}(\tau)\right), \Omega$, and $g$ are replaced by estimates satisfying conditions $A .4$ and $A . \%$.

The foregoing results provide some basic machinery for a broad class of tests based on the quantile regression process. In the next section we consider several special cases including tests of the location shift hypothesisTand tests for the location-scale shift hypothesis.

\section{IMPLEMENTATION OF THE TESTS}

Given a framework for inference based on the quantile regression process $\Gamma$ we can now -in a somewhat more pragmatic spirit- elaborate some missing details. We will begin by considering tests of the location scale shift hypothesis against a general quantile regression alternative. Tests of the location shift hypothesis and several variants of a symmetry hypothesis will then be considered. Problems associated with estimation of nuisance parameters are treated in the final subsection.

4.1. The location-scale shift hypothesis. We would like to test

$$
F_{y_{i} \mid x_{i}}^{-1}\left(\tau \mid x_{i}\right)=x_{i}^{\top} \alpha+x_{i}^{\top} \gamma F_{0}^{-1}(\tau)
$$

against the sequence of linear quantile regression alternatives

$$
F_{y_{i} \mid x_{i}}^{-1}\left(\tau \mid x_{i}\right)=x_{i}^{\top} \beta_{n}(\tau) .
$$

In the simplest case the univariate quantile function is known and we can formulate the hypothesis in the (3.4) notation $\Gamma$

$$
R \beta(\tau)-r=\Psi(\tau)
$$

by setting $r_{i}=\alpha_{i} / \gamma_{i}, \quad R=\operatorname{diag}\left(\gamma_{i}^{-1}\right)$ Tand $\Psi(\tau)=\iota_{p} F_{0}^{-1}(\tau)$. Obviously difficulty if there are $\gamma_{i}$ equal to zero. In such cases $\Gamma$ we can take $\gamma_{i}=1$ Tand set the 
corresponding elements $r_{i}=\alpha_{i}$ and $\Psi_{i}(\tau) \equiv 0$. How should we go about estimatng the parameters $\alpha$ and $\gamma$ ? Under the null hypothesis $\Gamma$

$$
\beta_{i}(\tau)=\alpha_{i}+\gamma_{i} F_{0}^{-1}(\tau) \quad i=1, \ldots, p
$$

so it is natural to consider linear regression. Since $\hat{\beta}_{i}(\tau)$ is piecewise constant with jumps at joints $\mathcal{J}=\left\{\tau_{1}, \ldots, \tau_{J}\right) \quad j=1, \ldots, J$. it suffices to consider $p$ bivariate linear regressions of $\hat{\beta}_{i}\left(\tau_{j}\right)$ on $\left\{\left(1, F_{0}^{-1}\left(\tau_{j}\right)\right): j=1, \ldots, J\right.$. Each of these regressions has a known (asymptotic) Gaussian covariance structure that could be used to construct a weighted least squares estimatorTbut pragmatism might lead us to opt for the simpler unweighted estimator. In either case we have our required $\mathcal{O}\left(n^{-1 / 2}\right)$ estimators $\hat{\alpha}_{n}$ and $\hat{\gamma}_{n}$.

When $F_{0}^{-1}(\tau)$ is (hypothetically) known the Khmaladzation process is relatively painless. The function $\dot{g}(t)=\left(1, \psi_{0}(t), \psi_{0}(t) F_{0}^{-1}(t)\right)^{\top}$ is known and the transformation (2.3) can be carried out by recursive least squares. Again $\Gamma$ the discretization is based on the jumps $\mathcal{J}=\left\{\tau_{1}, \ldots, \tau_{J}\right\}$ of the piecewise constant $\hat{\beta}(\tau)$ process. Tests statistics based on the transformed process $\Gamma \tilde{v}_{n}(\tau) \Gamma$ can then be easily computed. The simplest of these is probably the Kolmogorov-Smirnov sup-type statistic

$$
K_{n}=\sup _{\tau \in \mathcal{T}}\left\|\tilde{v}_{n}(\tau)\right\|
$$

where $\mathcal{T}$ is typically of the form $[\varepsilon, 1-\varepsilon]$ with $\varepsilon \in(0,1 / 2)$.

When $F_{0}^{-1}(t)$ isn't assumed to be known under the null it is convenient to choose one coordinate Ttypically the intercept coefficient Tto play the role of numeraire. From (3.4) we can write

$$
\beta_{i}(\tau)=\mu_{i}+\sigma_{i} \beta_{1}(\tau) \quad i=2, \ldots, p
$$

where $\mu_{i}=\alpha_{i}-\alpha_{1} \gamma_{i} / \gamma_{1}$ and $\sigma_{i}=\gamma_{i} / \gamma_{1}$ Tor in matrix notation as

$$
R \beta(\tau)=r
$$

where $\Psi(\tau) \equiv 0, \quad R=\left[\sigma:-I_{p-1}\right]$ and $r=-\mu$. Estimates of the vectors $\mu$ and $\sigma$ are again obtainable by regression of $\hat{\beta}_{i}(\tau) \quad i=2, \ldots, p-1$ on the intercept coordinate $\hat{\beta}_{1}(\tau)$.

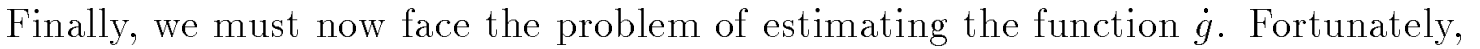
there is already a large literature on estimation of score functions. For our purposes it is convenient to employ the adaptive kernel method described in ?. An attractive alternative to this approach has been developed by ? and ? based on smoothing spline methods. Given a uniformly consistent estimator $\Gamma \dot{g}_{n} \Gamma$ satisfying condition A.7 $\Gamma$ see Portnoy and Koenker (1989 ГLemma 3.2) TCorollary 3 implies that under the null hypothesis

$$
\tilde{v}_{n}(t) \equiv Q_{g_{n}} \hat{v}_{n}(t) \Rightarrow w_{0}(t)
$$


and therefore Tests can be based on

$$
K_{n}=\sup _{\tau \in \mathcal{T}}\left\|\tilde{v}_{n}(\tau)\right\| .
$$

as before. Note that in this case estimation of $\dot{g}$ provides as a byproduct an estimator of the function $f_{0}\left(F_{0}^{-1}(t)\right)$ which is needed to compute the process $\hat{v}_{n}(t)$.

4.2. The location shift hypothesis. An important special case of the locationscale shift model is of course $\Gamma$ the pure location shift model $\Gamma$

$$
F_{y_{i} \mid x_{i}}^{-1}\left(\tau \mid x_{i}\right)=x_{i}^{\top} \alpha+\gamma F_{0}^{-1}(\tau)
$$

This is just the classical homescedastic linear regression model $\Gamma$

$$
y_{i}=x_{i}^{\top} \alpha+\gamma u_{i}
$$

where the $\left\{u_{i}\right\}$ are iid with distribution function $F_{0}$. This model underlies much of classical econometric theory and practice. If it is found to be appropriate then it is obviously sensible to consider estimation by alternative methods. For $F_{0}$ Gaussian $\Gamma$ least squares would of course be optimal. For $F_{0}$ unknown one might consider the Huber M-estimator Tor its L-estimator counterpart $\Gamma$

$$
\hat{\beta}_{\alpha}=(1-2 \alpha)^{2} \int_{\alpha}^{1-\alpha} \hat{\beta}(\tau) d \tau,
$$

see ?. In the location shift model it is also well-known from ? That the slope parameters $\Gamma\left(\beta_{2}, \ldots, \beta_{p}\right)$ Tare adaptively estimable provided $F_{0}$ has finite Fisher information for the location parameter. Thus Tit would be reasonable to consider M-estimators like those described in ? or the adaptive L-estimators described in ?.

The location-shift hypothesis can be expressed in standard form $\Gamma$

$$
R \beta(\tau)=r
$$

by setting $R=\left[0: I_{p-1}\right], r=\left(\alpha_{2}, \ldots, \alpha_{p}\right)^{\top}$. It asserts simply that the quantile regression slopes are constant $\Gamma$ independent of $\tau$. Again $\Gamma$ the unknown parameters in $\{R, r\}$ are easily estimated so the process $\hat{v}_{n}(\tau)$ is easily constructed. The transformation is obviously somewhat simpler in this case since $g(t)=\left(t, \varphi_{0}(t)\right)$ has one fewer coordinate than in the previous case.

We can continue to view tests of the location-shift hypothesis as tests against a general quantile regression alternative represented in (A.3) Tor we can also consider the behavior of the tests against a more specialized class of lcoation-scale shift alternatives for which

$$
\zeta(\tau)=\zeta_{0} F_{0}^{-1}(\tau)
$$

for some fixed vector $\zeta_{0} \in \mathbb{R}^{p-1}$. In the latter setting we have a test for parametric heteroscedasticity and we can compare the performance of our very general class of tests against alternative tests designed to be more narrowly focused on heteroscedastic alternatives. We will explore this in Section 9.z below. 
An optimal (invariant) test in the parametric setting may be based on optimal L-estimator of scale with weight function $\Gamma$

$$
\omega(\tau)=\left.\frac{d}{d x}\left(x f^{\prime} / f\right)\right|_{x=F_{0}^{-1}(\tau)},
$$

see e.g. ?. Thus $\Gamma$ for exampleTin the normal (Gaussian) $\operatorname{model} \Gamma F_{0}=\Phi \Gamma$ we would have $\omega(\tau)=\Phi^{-1}(\tau)$, so our estimator of $\zeta_{0}$ would be $\Gamma$

$$
\hat{\zeta}_{n}=\int_{0}^{1} \Phi^{-1}(\tau) \hat{\beta}(\tau) d \tau,
$$

and a test for heteroscedasticity could be based on the last $p-1$ coordinates of $\hat{\zeta}_{n}$. One way to interpret such tests is to view them as smoothly weighted linear combinations of the interquantile range tests for heteroscedasticity introduced in ?. Clearly in the case of the Gaussian weight function Textreme interquantile ranges get considerable weight Tso it may be prudent to consider Huberized versions of these tests that trim the influence of the tails. Alternatively Tone could consider weight functions explicitly designed for more heavy tailed distributions like the Cauchy $\Gamma$

$$
\omega(\tau)=2 \sin (2 \pi \tau)(\cos (2 \pi \tau)-1) .
$$

4.3. Local Asymptotic Power Comparison. In this section we compare the heteroscedasticity tests proposed above in an effort to evaluate the cost of considering a much more general class of semiparametric alternatives instead of the strictly parametric alternatives represented by the location scale shift model.

4.4. Estimation of Nuisance Parameters. Our proposed tests depend crucially on estimates of the quantile density and quantile score functions: $f\left(F^{-1}(\tau)\right) \Gamma$ and - $f^{\prime}\left(F^{-1}(\tau)\right) / f\left(F^{-1}(\tau)\right)$. Fortunately $\mathrm{Tthere}$ is a large related literature on estimating $f\left(F^{-1}(\tau)\right)$, including e.g. ? $\Gamma$ ? $\Gamma$ ? $\Gamma$ and ?. Following Siddiqui $\Gamma$ and noting that $\Gamma$ $d F^{-1}(t) / d t=\left(f\left(F^{-1}(t)\right)\right)^{-1}$ Tit is natural to use the estimator $\Gamma$

$$
f_{n}\left(F_{n}^{-1}(t)\right)=\frac{2 h_{n}}{F_{n}^{-1}\left(t+h_{n}\right)-F_{n}^{-1}\left(t-h_{n}\right)},
$$

where $F_{n}^{-1}(s)$ is an estimate of $F^{-1}(s)$ and $h_{n}$ is a bandwidth which tends to zero as $n \rightarrow \infty$.

One way of estimating $F^{-1}(s)$ is to use a variant of the empirical quantile function for the linear model proposed in ? $\Gamma$

$$
F_{n}^{-1}(s)=\frac{\widehat{\alpha}(s)-\widehat{\alpha}}{\widehat{\sigma}} .
$$

If we use (4.3) in the formula (4.2) Tthe density $f\left(F^{-1}(t)\right)$ can be estimated by

$$
f_{n}\left(F_{n}^{-1}(t)\right)=\frac{2 h_{n} \widehat{\sigma}}{\widehat{\alpha}\left(t+h_{n}\right)-\widehat{\alpha}\left(t-h_{n}\right)}
$$


and thus

$$
\frac{f_{n}\left(F_{n}^{-1}(t)\right)}{\widehat{\sigma}}=\frac{2 h_{n}}{\widehat{\alpha}\left(t+h_{n}\right)-\widehat{\alpha}\left(t-h_{n}\right)}
$$

can be used in constructing testing statistics. Smoothed estimators based on (4.4) may also be used.

\section{Monte Carlo Results}

We have conducted some limited Monte Carlo experiments to examine the finite sample performance of the proposed tests. In particular $\Gamma$ we examine the effectiveness of the martingale transformation based on the size and power properties of the tests. The following sample sizes were considered in our experiment: $n=100,200,300,400,500$. These sample sizes were chosen because they represent the most relevant range of sample sizes in empirical analyses.

First of all to investigate the effectiveness of the martingale transformation on quantile regression inference we examine the size and power properties of the infeasible version tests where the true density and score functions are used in the standardization and the martingale transformation. We start with the heteroskedasticity test. The data were generated from

$$
y_{i}=\alpha+\beta x_{i}+\sigma\left(x_{i}\right) u_{i},
$$

where $x_{i}$ and $u_{i}$ are iid $\mathcal{N}(0,1)$ random variates and are mutually independent $\Gamma \alpha=0$, and $\beta=1 . \sigma\left(x_{i}\right)=\gamma_{0}+\gamma_{1} x_{i} \Gamma \gamma_{0}=1$. We examined the empirical rejection rates of the test for different choices of sample sizes and $\gamma_{1}$ valuesTat $5 \%$ level of significance. In constructing the test $\Gamma$ we used the OLS estimator for $\widehat{\beta}$ Tand the truncation parameter value $\delta=0.05$ (i.e. $\mathcal{T}=[0.05,0.95]$ ). Since $x_{i}$ is a scalar the limiting null distribution of the test statistic is $\sup _{0.05 \leq \tau \leq 0.95}|W(\tau)|$. The $5 \%$ level critical value is 2.14. For the choices of the heteroskedasticity parameter $\gamma_{1}$, we consider $\gamma_{1}=0,0.1,0.2,0.3,0.5,1,2,5$. When $\gamma_{1}=0$, the model is homoskedastic and the rejection rates give the empirical sizes. When $\gamma_{1} \neq 0$, the model is heteroskedastic and the rejection rates deliver the empirical powers. Table 1 reports the empirical rejection rates for different values of $\gamma_{1}$ and $n$. Other values of the truncation parameter $\delta$ were also tried and quantitatively similar results were obtained. These Monte Carlo results indicate that given information on the density and score $\mathrm{t}$ the martingale transformation brings pretty good size and power to the proposed testing procedure in finite sample.

The remaining Monte-Carlo experiments are based on the even simpler two sample model $\Gamma$

$$
\left\{\begin{array}{l}
y_{1 i}=\alpha_{1}+\sigma_{1} u_{i}, i=1, \ldots . ., n_{1}, \\
y_{2 i}=\alpha_{2}+\sigma_{2} v_{i}, i=1, \ldots ., n_{2},
\end{array}\right.
$$


In particular Twe considered the following two sets of parameter values

$$
\begin{aligned}
\text { Location Shift: } & \alpha_{1}=1, \alpha_{2}=0, \sigma_{1}=\sigma_{2}=1, \\
\text { Location-Scale Shift: } & \alpha_{1}=1, \alpha_{2}=0, \sigma_{1}=2, \sigma_{2}=1,
\end{aligned}
$$

where $u_{i}, v_{i}$ are iid $\mathcal{N}(0,1)$ random variates. When the parameters take the first set of values $\Gamma(5.2)$ represents a pure location shift model. The null hypothesis of a shift model can be tested by the procedure given in Section 4.2. When the data is generated from the second set parameters $\Gamma(5.2)$ is a location-scale shift model. The location-scale hypothesis can be tested by the procedure given in Section 4.1. Table 2 reports the empirical size of these tests for different combinations of $n_{1}$ and $n_{2}$. We can see that the test has good size properties in finite samples. These Monte Carlo results $\Gamma$ together with the results on the heteroskedasticity test in Table $1 \Gamma$ confirm the effectiveness of the martingale transformation in quantile regression inference.

The above Monte Carlo experiments use the true density and score. It is obviously important to evaluate the effect of nonparametric nuisance parameter estimation on the performance of the proposed tests. In our next Monte Carlo experimentsTwe estimated $F^{-1}(s)$ using the empirical quantile function approach given by formula (4.3). For the density function Twe use procedure (4.4) as an estimator of $f\left(F^{-1}(s)\right.$ ). The quantile score process $\Gamma$ and thereby the function $g$ Tis estimated by the adaptive kernel estimator of Portnoy and Koenker (1989).

The kernel estimation procedures for these nuisance functions are nonparametric and therefore obviously entail choices of bandwidth values. Unsuitable bandwidth selection can produce poor estimates. However Tunder broad conditions on the convergence rate of the bandwidth parameters $\Gamma$ the nonparametric estimates are consistent and testing procedures using different bandwidth choices are (first order) asymptotically equivalent Talthough the finite sample performance of these tests can vary considerably with bandwidth choice. Extensive simulations have been conducted in the literature to show the importance of bandwidth choice on estimation and testing procedure that use nonparametric estimates.

It was anticipated that the estimation of $f\left(F^{-1}(s)\right)$ would exert important influence on the finite sample performance of our tests. This is confirmed in the simulations. For this reason Twe pay particular attention to the bandwidth choice in density estimation. Hall and Sheather (1988) suggested a bandwidth rule based on Edgeworth expansion for studentized quantiles. This bandwidth is of order $n^{-1 / 3}$ and we denote it as $h_{H S}$. Another bandwidth selection has been proposed by Bofinger (1975) is of order $n^{-1 / 5}$. We denote it by $h_{B}$. We have considered both of these bandwidth choices for our tests. In addition Tnotice that the Bofinger bandwidth is eventually much larger than the Hall and Sheather bandwidth $\Gamma$ we have also considered the following bandwidth choice which takes values between $h_{H S}$ and $h_{B}$, it is denoted as $h_{\theta} \Gamma h_{\theta}=\theta h_{B} \Gamma$ where $h_{B}$ is the Bofinger bandwidth and $\theta$ is a scalar. We report the results for the case 
$\theta=0.6$ here. The score function was estimated by the method of Portnoy and Koenker (1989) and we simply choose the Silverman (1986) bandwidth.

Tables $3 \mathrm{a} \Gamma 3 \mathrm{~b} \Gamma 3 \mathrm{c}$ report the Monte Carlo results for the heterskedasticity test with different bandwidth selections and Tables $4 \mathrm{a} \Gamma 4 \mathrm{~b} \Gamma 4 \mathrm{c}$ give the result of the locationscale test. The Monte Carlo evidence indicates that the bandwidth choice does have an important influence on the finite sample performance of these tests. It also shows that $\Gamma$ by choosing appropriate bandwidth $\Gamma$ the proposed tests have reasonable size and power properties. In general $\Gamma$ we found over-rejection when the Hall-Sheather bandwidth was used. For the other two bandwidth $\Gamma h_{\theta}$ and $h_{B} \Gamma$ the relative performance depend on which test we consider. For the heteroskedasticity test $\Gamma$ we found under-rejection when the Bofinger bandwidth was used. In this test Tat least for the model and the nonparametric methods used here $\Gamma$ the bandwidth choice $h_{\theta}$ provides pretty good finite sample performance. HoweverTfor the location-scale test $\Gamma h_{\theta}$ tends to over-reject and $h_{B}$ seems to be a relatively better bandwidth choice. To focus our attention on the effect of $f_{n}\left(F_{n}^{-1}(s)\right)$ Twe have also conducted Monte Carlo experiments where only the density function is estimated (and use the true score function) $\Gamma$ the Monte Carlo results reconfirmed our findings on the three bandwidth choices.

TABLE 1: Size and Power of the Heteroskedasticity Test (Truncated $\Gamma \delta=0.05$ )

\begin{tabular}{|c|c|c|c|c|c|c|c|c|}
\hline & Size & Power & & & & & & \\
\hline$n$ & $\gamma_{1}=0$ & $\gamma_{1}=0.1$ & $\gamma_{1}=0.2$ & $\gamma_{1}=0.3$ & $\gamma_{1}=0.5$ & $\gamma_{1}=1$ & $\gamma_{1}=2$ & $\gamma_{1}=5$ \\
\hline 100 & 0.006 & 0.134 & 0.377 & 0.729 & 0.974 & 0.981 & 0.990 & 0.999 \\
\hline 200 & 0.054 & 0.269 & 0.77 & 0.977 & 0.999 & 1.000 & 1.000 & 1.000 \\
\hline 300 & 0.052 & 0.383 & 0.931 & 1.000 & 1.000 & 1.000 & 1.000 & 1.000 \\
\hline 400 & 0.052 & 0.549 & 0.989 & 1.000 & 1.000 & 1.000 & 1.000 & 1.000 \\
\hline 500 & 0.052 & 0.616 & 1.000 & 1.000 & 1.000 & 1.000 & 1.000 & 1.000 \\
\hline
\end{tabular}

TABLE 2: Application to The Two-Sample Models

\begin{tabular}{lllllllllllll}
\hline \multicolumn{3}{l}{ Case 1: Location Shift } & \multicolumn{4}{c}{ Case 2: Location-Scale Shift } \\
\hline \multicolumn{2}{l}{$\alpha_{1}=1, \alpha_{2}=0, \sigma_{1}=\sigma_{2}=1$} & \multicolumn{4}{c}{$\alpha_{1}=1, \alpha_{2}=0, \sigma_{1}=2, \sigma_{2}=1$} \\
\hline$n_{1}$ & $n_{2}$ & size & $n_{1}$ & $n_{2}$ & size & $n_{1}$ & $n_{2}$ & size & $n_{1}$ & $n_{2}$ & size \\
\hline 100 & 100 & 0.074 & 100 & 200 & 0.060 & 100 & 100 & 0.153 & 100 & 200 & 0.179 \\
\hline 150 & 150 & 0.080 & 100 & 300 & 0.086 & 150 & 150 & 0.158 & 100 & 300 & 0.196 \\
\hline 200 & 200 & 0.064 & 150 & 300 & 0.055 & 200 & 200 & 0.169 & 150 & 300 & 0.175 \\
\hline 250 & 250 & 0.054 & 200 & 300 & 0.056 & 250 & 250 & 0.172 & 200 & 300 & 0.183 \\
\hline
\end{tabular}

(The Heteroskadasticity Test. Bandwidth in Density Estimation: $h_{H S}$; Kernel Estimation of Score with Silverman Bandwidth) 


\begin{tabular}{lllll}
\hline & Size & Power & & \\
\hline$n$ & $\gamma_{1}=0$ & $\gamma_{1}=0.2$ & $\gamma_{1}=0.5$ & $\gamma_{1}=1$ \\
\hline 100 & 0.45 & 0.723 & 0.99 & 1.000 \\
\hline 200 & 0.21 & 0.877 & 1.000 & 1.000 \\
\hline 300 & 0.195 & 0.952 & 1.000 & 1.000 \\
\hline 400 & 0.186 & 0.995 & 1.000 & 1.000 \\
\hline 500 & 0.173 & 1.000 & 1.000 & 1.000 \\
\hline
\end{tabular}

TABLE $3 \mathrm{~b}$

(The Heteroskadasticity Test. Bandwidth in Density Estimation: $h_{B}$;

Kernel Estimation of Score with Silverman Bandwidth)

\begin{tabular}{lllll}
\hline \multicolumn{3}{c}{ Size } & \multicolumn{3}{c}{ Power } \\
\hline$n$ & $\gamma_{1}=0$ & $\gamma_{1}=0.2$ & $\gamma_{1}=0.5$ & $\gamma_{1}=1$ \\
\hline 100 & 0.009 & 0.053 & 0.197 & 0.545 \\
\hline 200 & 0.013 & 0.109 & 0.772 & 0.949 \\
\hline 300 & 0.019 & 0.229 & 0.985 & 0.992 \\
\hline 400 & 0.023 & 0.412 & 0.997 & 0.998 \\
\hline 500 & 0.029 & 0.565 & 1.000 & 1.000 \\
\hline
\end{tabular}

TABLE $3 \mathrm{c}$

(The Heteroskadasticity Test. Bandwidth in Density Estimation: $h_{\theta}$; Kernel Estimation of Score with Silverman Bandwidth)

\begin{tabular}{lllll}
\hline \multicolumn{3}{c}{ Size } & \multicolumn{3}{c}{ Power } \\
\hline$n$ & $\gamma_{1}=0$ & $\gamma_{1}=0.2$ & $\gamma_{1}=0.5$ & $\gamma_{1}=1$ \\
\hline 100 & 0.035 & 0.211 & 0.755 & 0.820 \\
\hline 200 & 0.041 & 0.406 & 0.990 & 0.989 \\
\hline 300 & 0.043 & 0.665 & 1.000 & 1.000 \\
\hline 400 & 0.043 & 0.809 & 1.000 & 1.000 \\
\hline 500 & 0.045 & 0.911 & 1.000 & 1.000 \\
\hline \multicolumn{4}{c}{ TABLE 4a }
\end{tabular}

(Location-Scale Test. Bandwidth in Density Estimation: $h_{H S}$;

Kernel Estimation of Score with Silverman Bandwidth)

\begin{tabular}{llllll}
\hline$n_{1}$ & $n_{2}$ & size & $n_{1}$ & $n_{2}$ & size \\
\hline 100 & 100 & 0.589 & 50 & 50 & 0.616 \\
\hline 150 & 150 & 0.538 & 75 & 75 & 0.603 \\
\hline 200 & 200 & 0.511 & 250 & 250 & 0.507 \\
\hline 500 & 500 & 0.406 & 300 & 300 & 0.456 \\
\hline
\end{tabular}

TABLE $4 b$

(Location-Scale Test. Bandwidth in Density Estimation: $h_{B}$; Kernel Estimation of Score with Silverman Bandwidth) 


\begin{tabular}{llllll}
\hline$n_{1}$ & $n_{2}$ & size & $n_{1}$ & $n_{2}$ & size \\
\hline 100 & 100 & 0.037 & 50 & 50 & 0.028 \\
\hline 150 & 150 & 0.079 & 75 & 75 & 0.033 \\
\hline 200 & 200 & 0.079 & 250 & 250 & 0.065 \\
\hline 500 & 500 & 0.105 & 300 & 300 & 0.078 \\
\hline
\end{tabular}

TABLE $4 \mathrm{c}$

(Location-Scale Test. Bandwidth in Density Estimation: $h_{\theta}$; Kernel Estimation of Score with Silverman Bandwidth)

\begin{tabular}{llllll}
\hline$n_{1}$ & $n_{2}$ & size & $n_{1}$ & $n_{2}$ & size \\
\hline 100 & 100 & 0.097 & 50 & 50 & 0.063 \\
\hline 150 & 150 & 0.112 & 75 & 75 & 0.086 \\
\hline 200 & 200 & 0.123 & 250 & 250 & 0.126 \\
\hline 500 & 500 & 0.145 & 300 & 300 & 0.135 \\
\hline
\end{tabular}

\section{A Reappraisal of the Pennsylvania Reemployment Bonus EXPERIMENTS}

A common concern about unemployment insurance (UI) systems has been the suggestion that the insurance benefit acts as a disincentive for job-seekers and thus prolongs the duration of unemployment spells. During the 1980's several controlled experiments were conducted in the U.S. to test the incentive effects alternative compensation schemes for UI. In these experiments TUI claimants were offered a cash bonus if they found a job within some specified period of time and if the job was retained for a specified duration. The question addressed by the experiments was: would the promise of such a monetary lump-sum benefit provide a significant inducement for more intensive job-seeking and thus reduce the duration of unemployment?

In the first experiments conducted in Illinois a random sample of new UI claimants were told that they would receive a bonus of $\$ 500$ if they found full-time employment within 11 weeks after filing their initial claim Tand if they retained their new job for at least 4 months. These "treatment claimants" were then compared with a control group of claimants who followed the usual rules of the Illinois UI system. The Illinois experiment provided very encouraging initial indication of the incentive effects of such policies. They showed that bonus offers resulted in a significant reduction in the duration of unemployment spells and consequent reduction of the regular amounts paid by the state to UI beneficiaries. This finding led to further "bonus experiments" in the states of New JerseyC Pennsylvania and Washington with a variety of new treatment options. An excellent review of the experiments $\Gamma$ some general conclusions about their efficacy and a critique of their policy relevance can be found in ?. In this 
section we will focus more narrowly on a reanalysis of data from the Pennsylvania Reemployment Bonus Demonstration described in detail in ?.

The Pennsylvania experiments were conducted by the U.S. Department of Labor between July 1988 and October 1989. During the enrollment period Tclaimants who became unemployed and registered for unemployment benefits in one of the selected local offices throughout the state were randomly assigned either to a control group or one of six experimental treatment groups. In the control group the existing rules of the unemployment insurance system applied. Individuals in the treatment groups were offered a cash bonus if they became reemployed in a full-time job Tworking more than 32 hours per week Twithin a specified qualification period. Two bonus levels and two qualification periods were tested Cbut we will restrict attention to the high bonus $\Gamma$ long qualification period treatment which offered a cash of bonus of six times the weekly benefit for claimants establishing reemployment within 12 weeks. A detailed description of the characteristics of claimants under study is presented in ? which has information on age $\Gamma$ race $\Gamma$ gender $\Gamma$ number of dependents $\Gamma$ location in the state $\Gamma$ existence of recall expectations Tand type of occupation.

Categorical variables related to these characteristics are used in our modeling. More specifically these are:

Treatment: indicator variable taking the value 1 if the claimant is in the treatment group and zero otherwise.

young: 1 if the claimant's age is less than 35 and 0 otherwise.

old: 1 if the claimant's age is more than 54 and 0 otherwise.

black: 1 if the claimant is black and 0 otherwise.

hispanic: 1 if the claimant is hispanic and 0 otherwise.

female: 1 if the claimant is female and 0 otherwise.

recall: 1 if the claimant answered "yes" when asked if he/she had any expectation to be recalled to his/her prior job.

dependents: indicates the number of dependents of the claimant. Coded $0 \Gamma 1$ Tor 2 if the number of dependents is 2 or greater.

lusd: 1 if the claimant filed in CoatesvilleTReading Tor Lancaster and 0 otherwise. These three sites were considered to be characterized by low unemployment rate and therefore shorter durations of unemployment.

durable: 1 if the occupation of the claimant was in the sector of durable manufacturing and 0 otherwise.

Q1-Q5: five indicator variables indicating the quarter of enrollment of each claimant.

Our measure of duration is called inuidur in the final reports of the experiment. Since a large portion of spells end in either the first or the twenty seventh week $\Gamma$ 
it should be stressed that the definition of the first spell of UI in the Pennsylvania study includes a waiting week and that the maximum number of uninterruptedly received full weekly benefits is 26 . This implies that many subjects did not receive any weekly benefit and that many other claimants received continuously their full $\Gamma$ entitled unemployment benefit. Again $\Gamma$ ? contains further details.

6.1. The Model. Our basic model for analyzing the Pennsylvania experiment presumes that the logarithm of the duration (in weeks) of subjects' spells of $U I$ benefits have linear conditional quantile functions of the form

$$
Q_{\log (T)}(\tau \mid x)=x^{\prime} \beta(\tau) \text {. }
$$

The choice of the $\log$ transformation is dictated primary by the desire to achieve linearity of the parametric specification and by its ease of interpretation. Multiplicative covariate effects are widely employed throughout survival analysisTand they are certainly more plausible in the present application than the assumption of additive effects. It is perhaps worth reiterating that the role of the transformation is completely transparent in the quantile regression setting $\Gamma$ where

$$
Q_{h(T)}(\tau \mid x)=x^{\prime} \beta(\tau)
$$

implies

$$
Q_{T}(\tau \mid x)=h^{-1}\left(x^{\prime} \beta(\tau)\right) .
$$

In contrast $\Gamma$ the role of transformations in models of the conditional mean function are rather complicated since the transformation affects not only location $\Gamma$ but scale and shape of the conditional distribution of the response. Our (provisional) model includes the following effects:

- Indicators for the treatment group.

- Indicators for female

- Number of dependents with 2 indicating two or more dependents.

- Indicators for the 5 quarters of entry to the experiment.

- Indicator for whether the claimant "expected to be recalled".

- Indicators for whether the respondent was "young" - less than 35Tor "old" greater than 54 .

- Indicator for whether claimant was employed in the durable goods industry.

- Indicator for whether the claimant was registered in a low employment district: CoatesvilleTReading Tor Lancaster.

In Figure 6.1 we present a concise visual representation of the results from the estimation of this model. Each of the panels of the Figure illustrate one coordinate of the vector-valued function $\Gamma \hat{\beta}(\tau) \Gamma$ viewed as a function of $\tau \in[\alpha, 1-\alpha]$. Here we choose $\alpha$ to be .20 effectively neglecting the proportion of the sample that are immediately reemployed in week one and those whose unemployment spell exceeds 
that insured limit of 26 weeks. The lightly shaded region in each panel of the figure represents a 90 percent confidence band.

Before turning to interpretation of specific coefficients $\Gamma$ we will try to offer some brief general remarks on how to interpret these figures. The simplest case is the pure location shift model in which we would have the classical accelerated failure time (AFT) model $\Gamma$

$$
\log T_{i}=x_{i}^{\prime} \beta+u_{i}
$$

with $\left\{u_{i}\right\}$ 's iid from some $F$. For $F$ of the form $F(u)=1-\exp (-\exp (u)) \Gamma$ this is the well known Cox proportional hazard model with Weibull baseline hazard. In this case we would expect to see coefficients $\hat{\beta}_{j}(\tau)$ that oscillate around a constant value indicating that the shift due to a change in the covariate is constant over the entire estimated range of the distribution.

Another conventional model with linear quantile functions is the linear locationscale model $\Gamma$

$$
\log T_{i}=x_{i}^{\prime} \beta+\left(x_{i}^{\prime} \gamma\right) u_{i}
$$

where again $\Gamma u_{i}$ is taken to be iid. Now the covariates are allowed to influence the scale as well as the location of the conditional distribution of durations. In this case the "slope" coefficients $\hat{\beta}_{j}(\tau)$ should look just like the "intercept" coefficient up to a location and scale shift. The intercept coefficient estimates a normalized version of the quantile function of the $u_{i}$ 's and all the other coefficients are simply location and scale shifts of this function.

No treatment effect is observed in either tail implying that the treatment had no effect in changing the probability of immediate reemployment (in week one) Tor in effecting the probability of durations beyond the 26 week maximum. The high bonus and long qualification period treatment yielded roughly a $15 \%$ reduction in median duration. This effect is considerably stronger statistical significance than that seen in the other treatments.

The randomization of the experiment was quite effective in rendering the potentially confounding effects of other covariates orthogonal to the treatment indicators. Nevertheless Tit is of some interest to explore the effect of other covariates in an effort to better understand determinants of the duration of unemployment.

Women are 5 to $15 \%$ slower than men to exit unemployment. Blacks and Hispanics appear much quicker than whites to become reemployed. This effect is particularly striking in the case of blacks for whom median duration is roughly half $\left(\approx e^{-.75}\right)$ that of whites $\Gamma$ and only $30 \%$ as long as controls at $\tau=.33$. The number of dependents appears to exert a rather weak positive effect on unemployment durations. The quarter-of-entry variables are inherently not very interesting Tbut it appears that late entry into the experiment improved one's chances for early reemployment. The recall indicator is considerably more interesting; anticipated recall to one's prior job has a very strong and very precisely estimated detrimental effect over the entire lower tail 
Figure 6.1. Quantile Regression Process for Log Duration Model
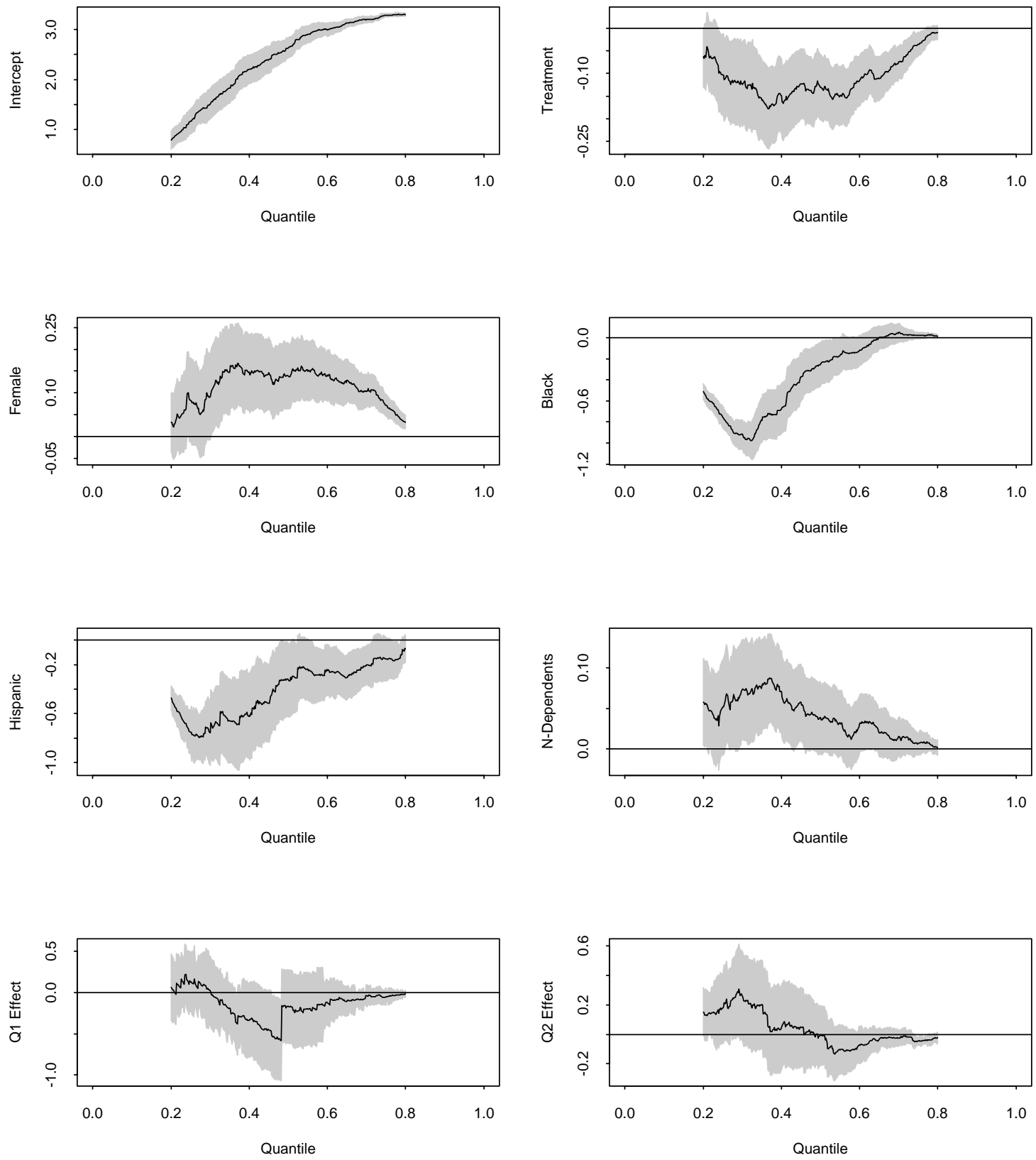

of the distribution. However Tbeyond quantile $\tau=.6$, which corresponds to about 20 weeks duration for whiteTmale controls $\Gamma$ the anticipated recall appears to be foresaken 

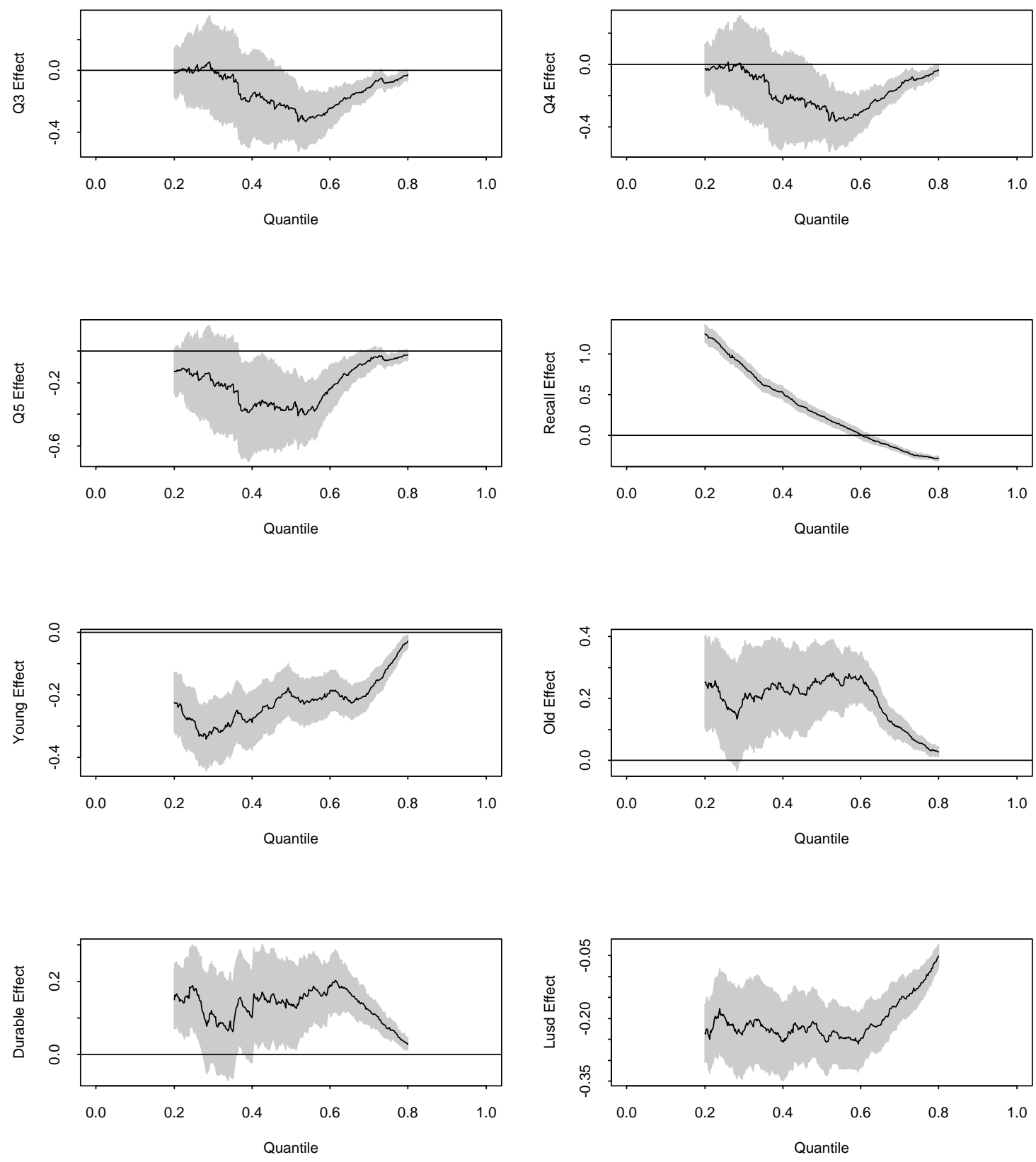

and beyond this point recall becomes a significant force for early reemployment in the upper tail of the distribution.

Not surprisingly the young (those under 34) tend to find reemployment earlier than their middle aged counterparts $\Gamma$ while the old (those over 54) do significantly 
worse. In both cases the effects are highly significant throughout the entire range of quantiles we have estimated. Prior employment in durable manufacturing has a weakly disadvantageous effect on reemployment Tbut residing in a low unemployment district is not surprisingly

The treatment effect of the bonus offer clearly does not conform to the location shift paradigm of the conventional models. After the $\log$ transformation of durations $\Gamma$ a location shift would imply that the treatment exerts a constant percentage change in all durations. In the present instance this implication is particularly unpalatable since the entire point of the experiment was to alter the shape of the conditional duration distribution. In the treatment panel of Figure 6.1 we have seen that the bonus effect gradually reduces durationsfrom a null effect in the lower tail to a maximum reduction of $15 \%$ at the median $\Gamma$ and then gradually again returns to a null effect in the upper tail. This finding accords perfectly with the timing imposed by the qualification period of the experiment. It might be thought that the bonus should not effect durations at all beyond the qualification period $\Gamma$ but further consideration suggests that accelerated search in an effort to meet the qualification period deadline could easily yield "successes" that extended beyond the qualification period due to decision delay by potential employers Tor other factors.

Taken together $\Gamma$ the results presented in Figure 6.1 do not seem to lend much support to either the location shift $\Gamma$ or to the location-scale shift $\Gamma$ hypotheses of the conventional regression model. In the former case we would expect to see plots that appeared essentially constant in $\tau$ while in the latter $\Gamma$ we expect to see plots that mimic the shape of the intercept plot. Neither of these expectations are fullfilled. However $\Gamma$ as we have emphasized earlier $\Gamma$ it is crucial to to be able evaluate these impressions by more formal statistical methods.

6.2. Inference on the Quantile Regression Process. To illustrate our proposed inference strategy we have decomposed the test of the location scale shift hypothesis based on the full model represented in Figure 6.1Tinto several intermediate steps. In each of these steps we present results for only a subset of eight selected covariate effects in an effort to conserve space $\Gamma$ but all 15 covariate effects are handled in an identical fashion. In Figure 6.2 we present $\Gamma$ for each of our selected covariates $\Gamma$ the prediction of the process $\hat{\beta}_{i}(\tau)$ based on the regression onto the estimated "intercept process" $\Gamma \hat{\beta}_{1}(\tau)$ as indicated by (4.1). Each of the fitted curves is based on least squares estimation using the 301 estimated points of the quantile regression process for each coordinate. The solid lines in these panels are the same as those appearing in the previous figure; the dotted lines represents the fitted curve. With the possible exception of the recall effect $\Gamma$ none of these fits look very compelling Tbut at this stage we are already deeply mired in the Durbin problem and so it is difficult to judge the significance of departures from the fitted relationships.

Taking the residuals from the panels of Figure $6.2 \Gamma$ and standardizing by the Cholesky decomposition of their (inverse) covariance matrix yields the parametric 
Figure 6.2. Quantile Regression Process for Log Duration Model
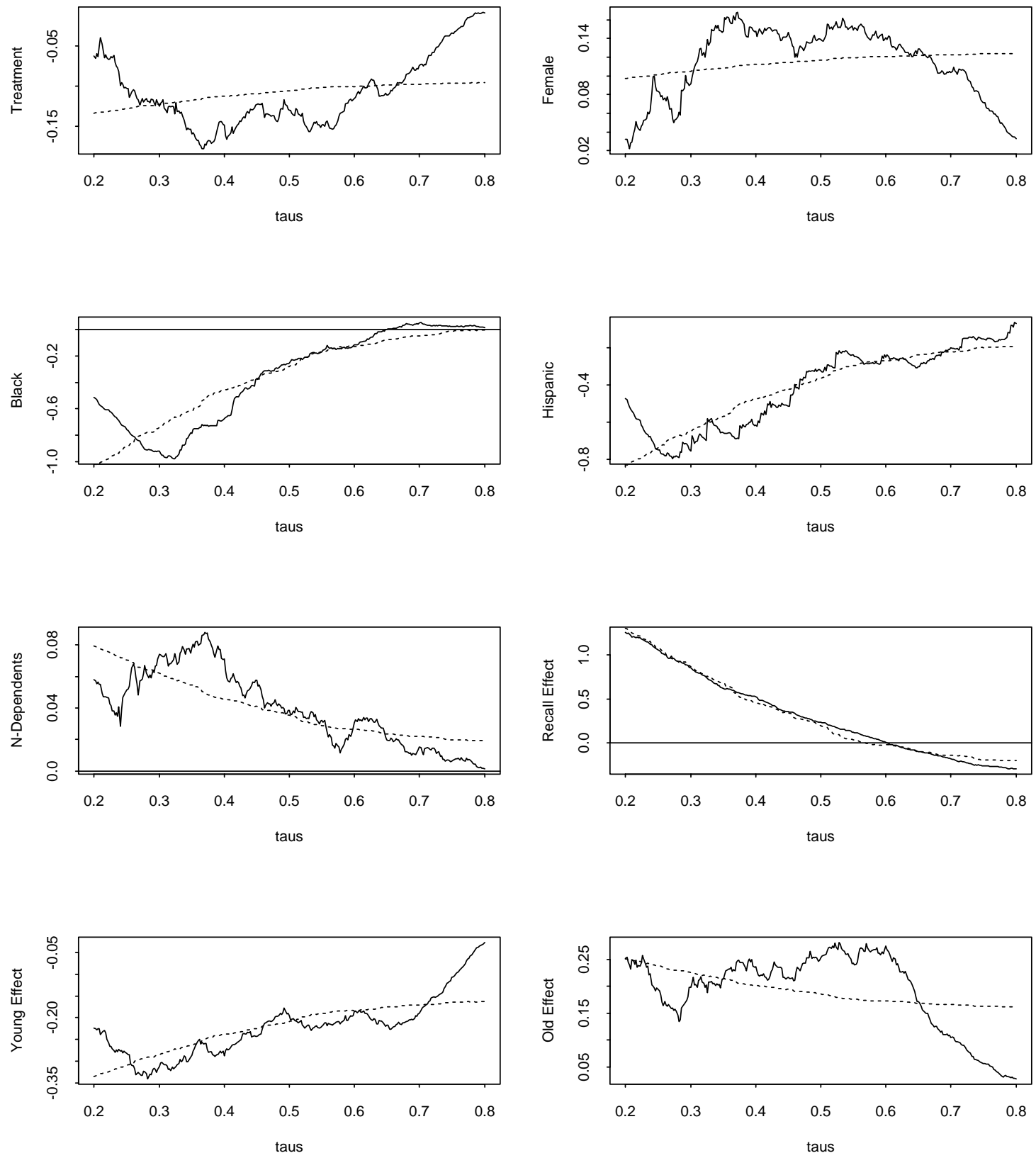

quantile regression process $\Gamma \hat{v}_{n}(\tau)$ Twhose coordinates are illustrated in Figure 6.3. It is perhaps misleading to associate the coordinates of this process so closely with the 
Figure 6.3. Parametric Quantile Regression Process for Log Duration Model
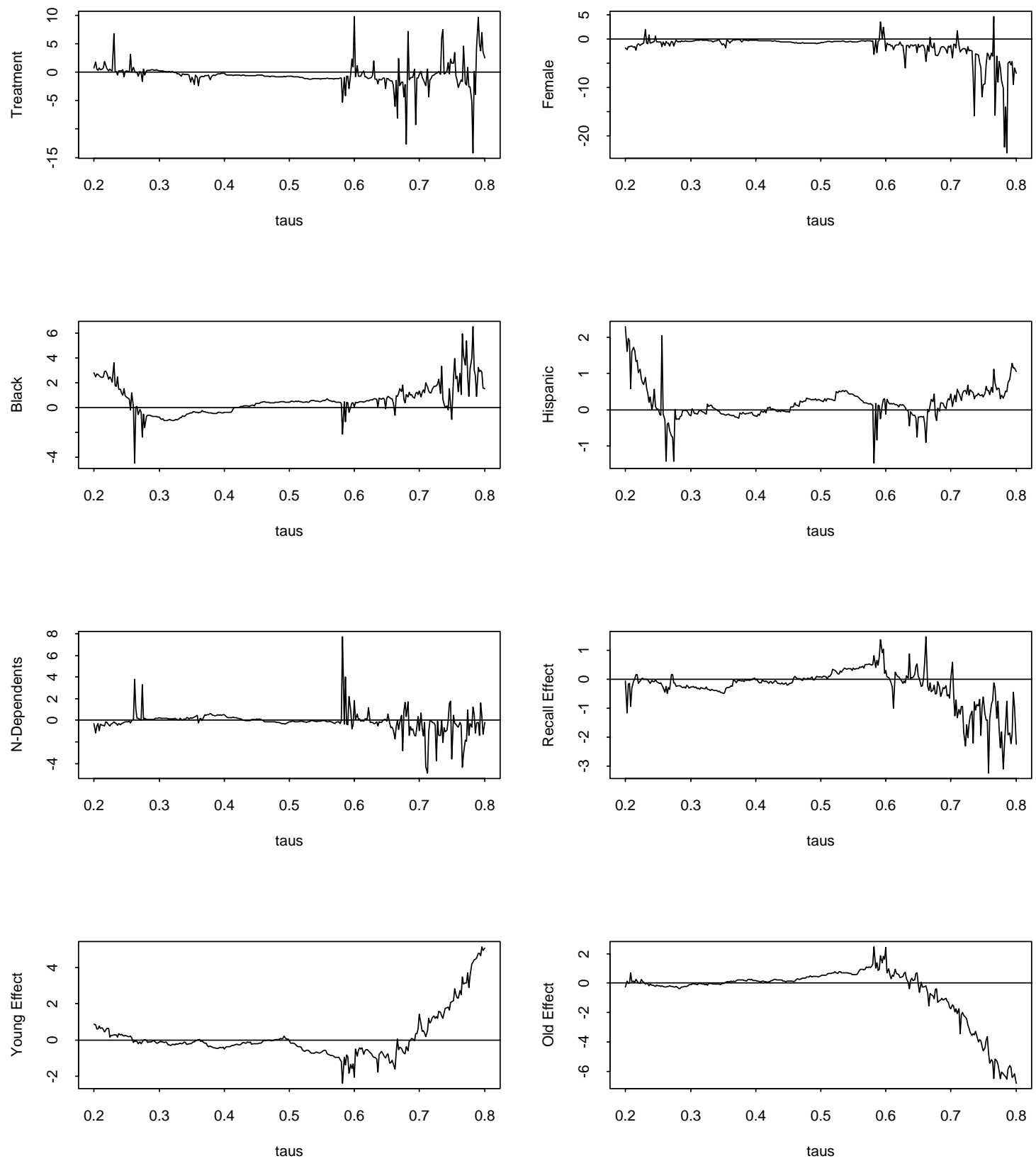

original labeling of the coordinates of $\hat{\beta}(\tau) \Gamma$ since the matrix transformation of the process mixes the coordinates thoroughly. Had we specified hypothetical values for 
Figure 6.4. Transformed Parametric Quantile Regression Process
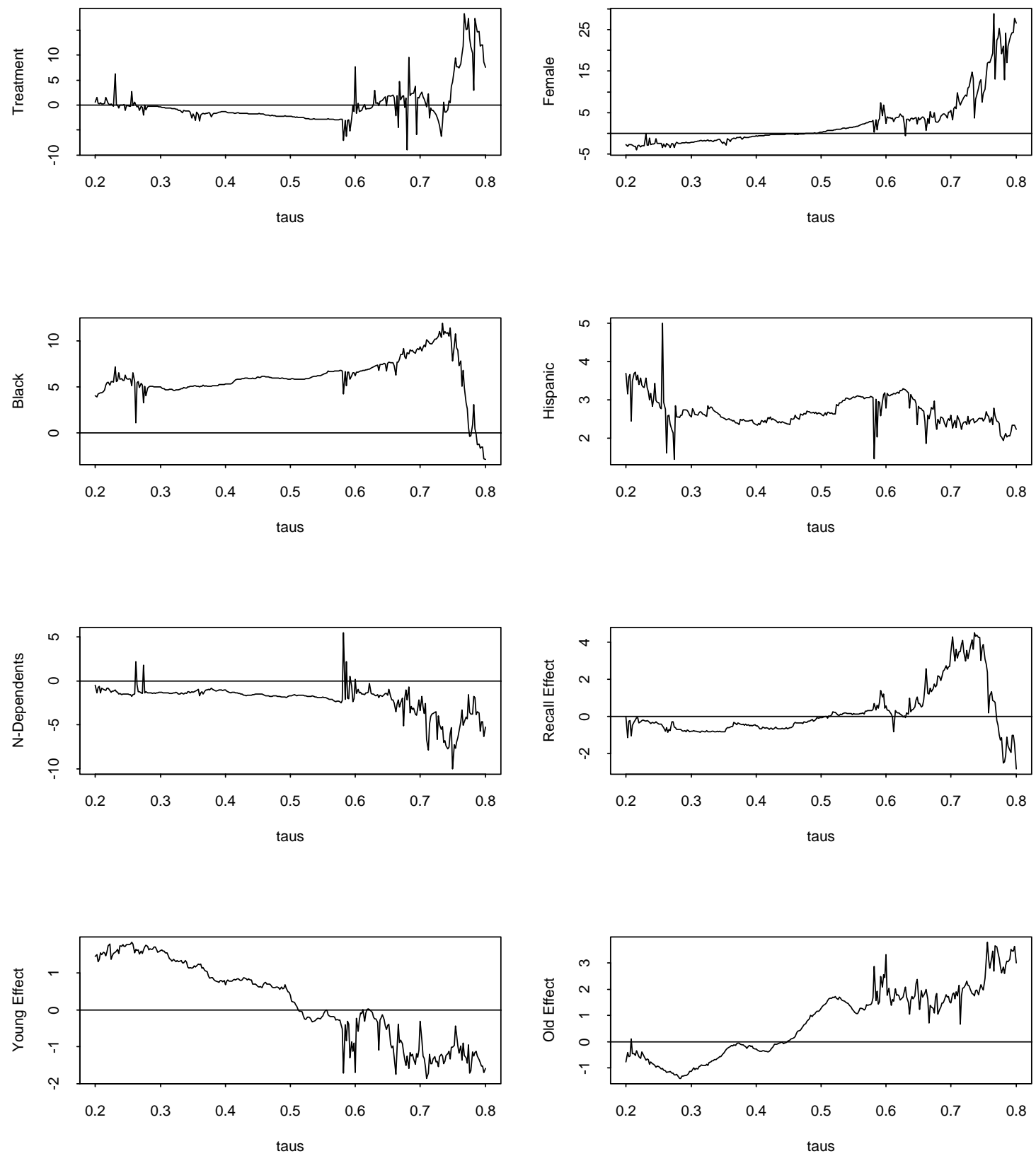

the coefficients rather than estimating them for Figure 6.2 Twe could of course treat the resulting process in Figure 6.3 as a vector of independent Brownian bridges under 
Figure 6.5. Parametric Quantile Regression Process
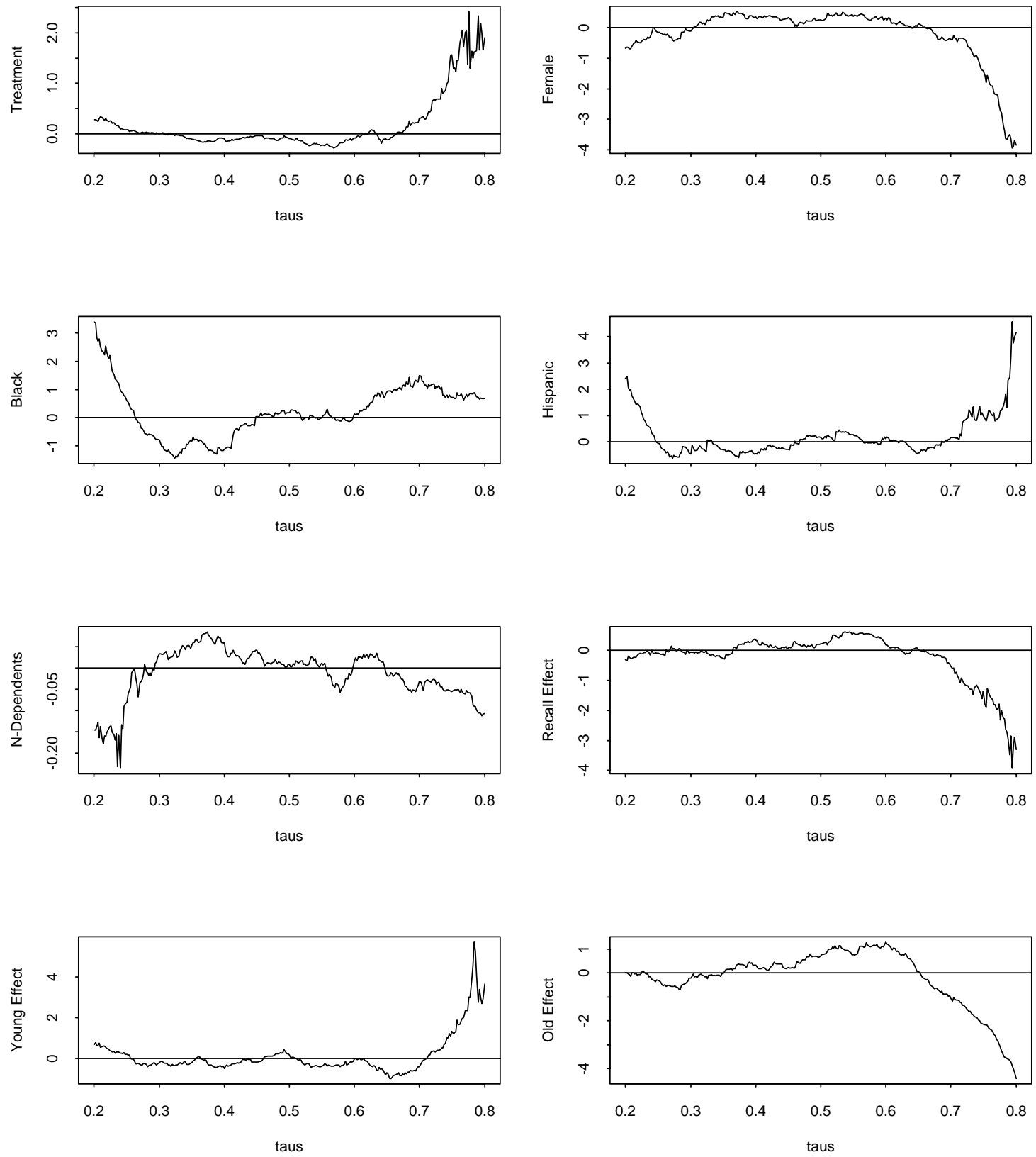

the null. However The effect of the estimation is to distort the variability of the process $\Gamma$ as we have seen in Section 3. At this point we estimate the function $\dot{g}$ and 
Figure 6.6. Transformed Parametric Quantile Regression Process
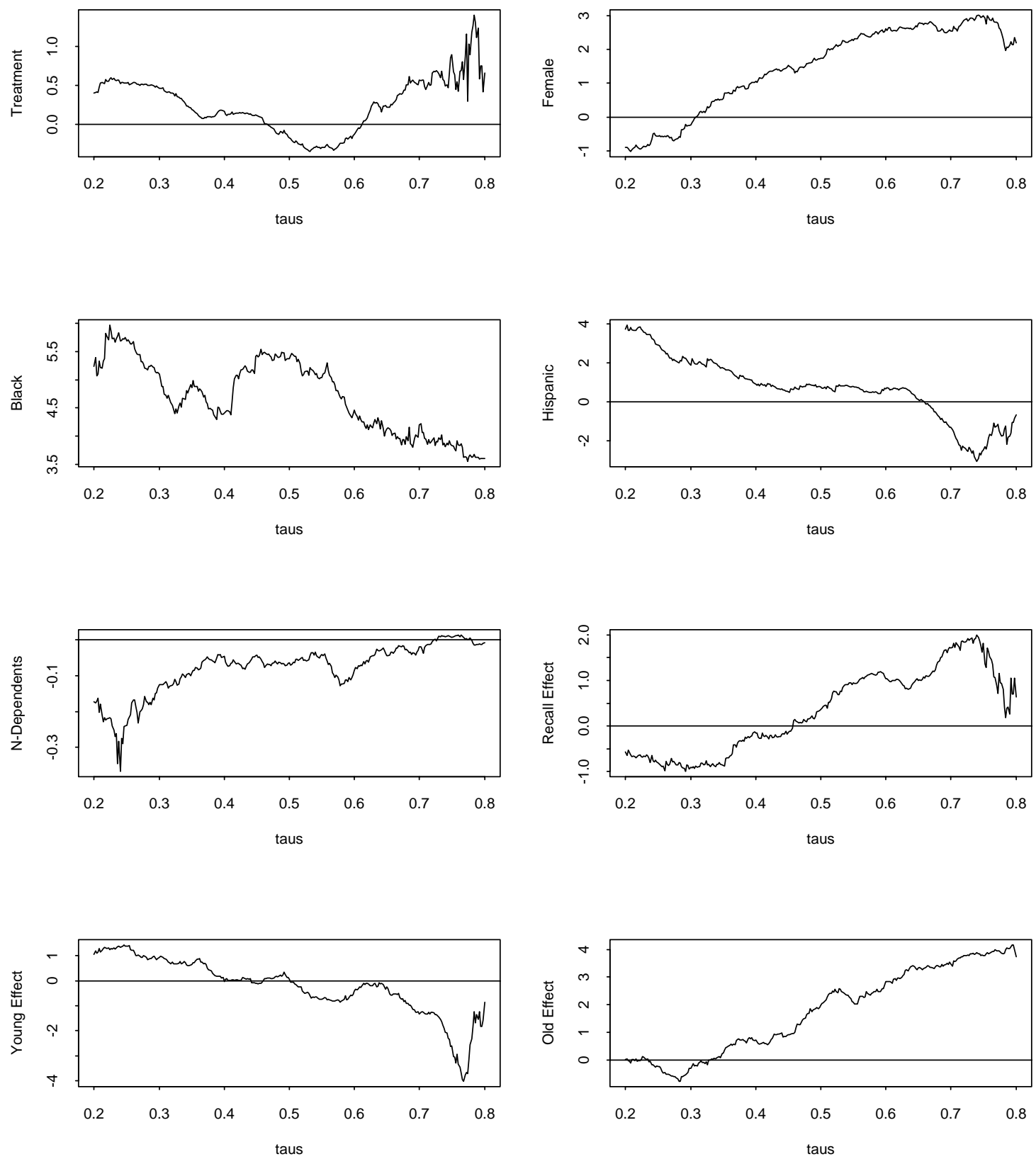

perform the martingale transformation on each slope coordinate. The transformed 
TABLE 6.1. $K_{n i}$ Statistics for the Log Duration Model

\begin{tabular}{|ll|ll|lr|}
\hline Treatment & 1.40 & Q1-Effect & 3.65 & Recall Effect & 1.99 \\
Female & 3.02 & Q2-Effect & 0.54 & Young Effect & 1.43 \\
Black & 5.97 & Q3-Effect & 0.16 & Old Effect & 4.16 \\
Hispanic & 3.93 & Q4-Effect & 0.25 & Durable Effect & 1.72 \\
N-Dependents & 0.01 & Q5-Effect & 0.20 & Lusd Effect & -2.23 \\
\hline
\end{tabular}

coordinates of the process $\tilde{v}_{n}(\tau)$ Tare illustrated in Figure 6.4 . Under the null hypoth-

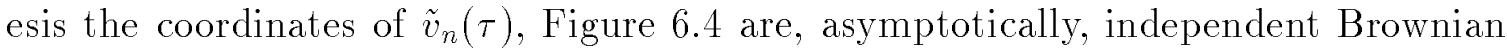
motions. We consider the test statistic $\Gamma$

$$
K_{n}=\sup _{\tau \in \mathcal{T}}\left\|\tilde{v}_{n}(\tau)\right\|_{1}
$$

which takes the value 114.78 . The critical value for this test is 16.55 S so the locationscale-shift hypothesis is decisively rejected.

It is of some independent interest to investigate which of the coordinates contribute most to the joint significance of our $K_{n}$ statistic. This inquiry is fraught with all the usual objections Tbut we plunge ahead. In place of the joint hypothesis we can consider univariate sub-hypotheses of the form $\Gamma$

$$
\beta_{i}(\tau)=\mu_{i}+\sigma_{i} \beta_{1}(\tau)
$$

for each "slope" coefficient. In effect this approach replaces the matrix standardization used for the joint test by a scalar standardization. The martingale transformation is then applied just as in the previous case. Now Tbecause there is no matrix standardization the original labeling of the coordinates is more meaningful. In Figure 6.5 we replot the standardized residuals for our eight selected covariate effects using this coordinatewise approach. And in Figure 6.6 we plot these processes after the martingale transformation. In Table 6.1 we present the the test statistics $\Gamma$

$$
K_{n i}=\sup _{\tau \in \mathcal{T}}\left|\tilde{v}_{n i}(\tau)\right|
$$

for each of the covariates. Effects for the quarter of entry are not reported. The critical values for these coordinatewise tests are given in Appendix $\mathrm{B} \Gamma$ and we see that with the exception of the dependent effect Tall the effects are quite highly significant.

What should we conclude from this exercise? The linear location shift and locatioscale shift models are very elegant and convenient abstractions for many statistical purposes. However Tthey also clearly place very strigent restrictions on the way that covariates are permitted to influence the conditional distribution of the response variable. In the case of our unemployment duration application the location-scale shift hypothesis may be viewed as a generalized form of the familiar accelerated failure time model in which the scale of the response distribution responds linearly to the covariates. This specification is decisively rejected by the data from the Pennsylvania 
experiments. Not only the treatment effect of the bonus payment Tbut many other of the covariates appear to affect the conditional distribution of unemployment duration in ways that are not adequately represented by pure location and/or scale shifts. One consequence of the proposed methods of inferencelit may be hoped $\Gamma$ would be a greater willingness to explore more flexible models for covariate effects.

\section{Appendix A.}

Proof of Theorem 1 Notice that

$$
R \hat{\beta}(\tau)-r-\Psi(\tau)=R[\hat{\beta}(\tau)-\beta(\tau)]+R \beta(\tau)-r-\Psi(\tau) .
$$

Under Assumption A.3, $R \beta(\tau)-r-\Psi(\tau)=\zeta(\tau) / \sqrt{n}$, thus

$$
R \widehat{\beta}(\tau)-r-\Psi(\tau)=R[\hat{\beta}(\tau)-\beta(\tau)]+\zeta(\tau) / \sqrt{n}
$$

Under Assumptions A.1 and A.2, by Theorem 1 of Gutenbrunner and Jureckova (1992), we have, uniformly for $\tau \in \mathcal{T}$,

$$
\sqrt{n}[\widehat{\beta}(\tau)-\beta(\tau)] \Rightarrow \frac{1}{\varphi(\tau)} H_{0}^{-1} J_{0}^{1 / 2} v_{0}(\tau)
$$

where $v_{0}(\tau)$ is a standardized $p$-dimensional Brownian bridge process, $\varphi(\tau)=f\left(F^{-1}(\tau)\right)$. Thus

$$
\begin{aligned}
v_{n}(\tau) & =\sqrt{n} \varphi(\tau)\left[R \Omega R^{\top}\right]^{-1 / 2}[R \hat{\beta}(\tau)-r-\Psi(\tau)] \\
& =\varphi(\tau)\left[R \Omega R^{\top}\right]^{-1 / 2} R \sqrt{n}[\hat{\beta}(\tau)-\beta(\tau)]+\varphi(\tau)\left[R \Omega R^{\top}\right]^{-1 / 2} \zeta(\tau) \\
& \Rightarrow v_{0}(\tau)+\eta(\tau)
\end{aligned}
$$

Proof of Corollary 1

$$
\begin{aligned}
v_{n}(\tau)= & \sqrt{n} \varphi_{n}(\tau)\left[R \Omega_{n} R^{\top}\right]^{-1 / 2}[R \hat{\beta}(\tau)-r-\Psi(\tau)] \\
= & \sqrt{n} \varphi(\tau)\left[R \Omega R^{\top}\right]^{-1 / 2}[R \widehat{\beta}(\tau)-r-\Psi(\tau)] \\
& +\left[\varphi_{n}(\tau)-\varphi(\tau)\right]\left[R \Omega_{n} R^{\top}\right]^{-1 / 2} \sqrt{n}[R \widehat{\beta}(\tau)-r-\Psi(\tau)] \\
& +\varphi(\tau)\left[\left[R \Omega_{n} R^{\top}\right]^{-1 / 2}-\left[R \Omega R^{\top}\right]^{-1 / 2}\right] \sqrt{n}[R \widehat{\beta}(\tau)-r-\Psi(\tau)]
\end{aligned}
$$

Notice that

$$
\left[R \Omega_{n} R^{\top}\right]^{-1 / 2}-\left[R \Omega R^{\top}\right]^{-1 / 2}=\left[R \Omega_{n} R^{\top}\right]^{-1 / 2}\left\{\left[R \Omega R^{\top}\right]^{1 / 2}-\left[R \Omega_{n} R^{\top}\right]^{1 / 2}\right\}\left[R \Omega R^{\top}\right]^{-1 / 2},
$$

and $\left[R \Omega_{n} R^{\top}\right]^{1 / 2}=R \hat{H}_{0}^{-1} J_{0}^{1 / 2}$,

$$
\left[R \Omega R^{\top}\right]^{1 / 2}-\left[R \Omega_{n} R^{\top}\right]^{1 / 2}=R\left[H_{0}^{-1}-\widehat{H}_{0}^{-1}\right] J^{1 / 2}=R \widehat{H}_{0}^{-1}\left[\widehat{H}_{0}-H_{0}\right] H_{0}^{-1} J^{1 / 2} .
$$

Under Assumption A.4,

$$
\begin{aligned}
& {\left[\varphi_{n}(\tau)-\varphi(\tau)\right]\left[R \Omega_{n} R^{\top}\right]^{-1 / 2} \sqrt{n}[R \hat{\beta}(\tau)-r-\Psi(\tau)]=o_{p}(1),} \\
& \varphi(\tau)\left[\left[R \Omega_{n} R^{\top}\right]^{-1 / 2}-\left[R \Omega R^{\top}\right]^{-1 / 2}\right] \sqrt{n}[R \widehat{\beta}(\tau)-r-\Psi(\tau)]=o_{p}(1),
\end{aligned}
$$


thus

$$
\begin{aligned}
v_{n}(\tau) & =\sqrt{n} \varphi_{n}(\tau)\left[R \Omega_{n} R^{\top}\right]^{-1 / 2}[R \widehat{\beta}(\tau)-r-\Psi(\tau)] \\
& =\sqrt{n} \varphi(\tau)\left[R \Omega R^{\top}\right]^{-1 / 2}[R \widehat{\beta}(\tau)-r-\Psi(\tau)]+o_{p}(1) \\
& \Rightarrow v_{0}(\tau)+\eta(\tau) .
\end{aligned}
$$

\section{Proof of Theorem 2}

$$
\begin{aligned}
\widehat{v}_{n}(\tau)= & \sqrt{n} \varphi(\tau)\left[R_{n} \Omega R_{n}^{\top}\right]^{-1 / 2}\left[R_{n} \widehat{\beta}(\tau)-r_{n}-\Psi(\tau)\right] \\
= & \varphi(\tau)\left[R_{n} \Omega R_{n}^{\top}\right]^{-1 / 2} \sqrt{n}[R \widehat{\beta}(\tau)-r-\Psi(\tau)] \\
& +\varphi(\tau)\left[R_{n} \Omega R_{n}^{\top}\right]^{-1 / 2} \sqrt{n}\left[r_{n}-r\right]+\varphi(\tau)\left[R_{n} \Omega R_{n}^{\top}\right]^{-1 / 2} \sqrt{n}\left[R_{n}-R\right] \widehat{\beta}(\tau) \\
= & \varphi(\tau)\left[R \Omega R^{\top}\right]^{-1 / 2} \sqrt{n}[R \widehat{\beta}(\tau)-r-\Psi(\tau)] \\
& +\varphi(\tau)\left[R \Omega R^{\top}\right]^{-1 / 2} \sqrt{n}\left[r_{n}-r\right]+\varphi(\tau) \beta(\tau)\left[R \Omega R^{\top}\right]^{-1 / 2} \sqrt{n}\left[R_{n}-R\right] \\
& +o_{p}(1)
\end{aligned}
$$

Notice that $\beta(\tau)=\alpha+\gamma F^{-1}(\tau)$,

$$
\begin{aligned}
\widehat{v}_{n}(\tau)= & \varphi(\tau)\left[R \Omega R^{\top}\right]^{-1 / 2} \sqrt{n}[R \widehat{\beta}(\tau)-r-\Psi(\tau)] \\
& +\varphi(\tau)\left\{\left[R \Omega R^{\top}\right]^{-1 / 2} \sqrt{n}\left[r_{n}-r\right]+\alpha\left[R \Omega R^{\top}\right]^{-1 / 2} \sqrt{n}\left[R_{n}-R\right]\right\} \\
& +\varphi(\tau) F^{-1}(\tau) \gamma\left[R \Omega R^{\top}\right]^{-1 / 2} \sqrt{n}\left[R_{n}-R\right] \\
& +o_{p}(1) \\
= & v_{n}(\tau)+\xi(\tau)^{\top} Z_{n}+o_{p}(1)
\end{aligned}
$$

where

$$
\xi(\tau)=\left(\varphi(\tau), \varphi(\tau) F^{-1}(\tau)\right)^{\top}
$$

and

$$
Z_{n}=\left[\begin{array}{l}
{\left[R \Omega R^{\top}\right]^{-1 / 2} \sqrt{n}\left[r_{n}-r\right]+\alpha\left[R \Omega R^{\top}\right]^{-1 / 2} \sqrt{n}\left[R_{n}-R\right]} \\
\gamma\left[R \Omega R^{\top}\right]^{-1 / 2} \sqrt{n}\left[R_{n}-R\right]
\end{array}\right]=O_{p}(1) .
$$

By result of Theorem 1,

$$
\widehat{v}_{n}(\tau)-\xi(\tau)^{\top} Z_{n} \Rightarrow v_{0}(\tau)+\eta(\tau)
$$

\section{Proof of Corollary 2}

Similar to that of Corollary 1.

\section{Proof of Theorem 3}

By the result of Theorem 2,

$$
\widehat{v}_{n}(\tau)=v_{0}(\tau)+\xi(\tau)^{\top} Z_{n}+\eta(\tau)+o_{p}(1) .
$$

Denote the transformation based on $\dot{g}$ as

$$
Q_{g}(h(\tau))=h(\tau)-\int_{0}^{\tau}\left[\dot{g}(s)^{\top} C(s)^{-1} \int_{s}^{1} \dot{g}(r) d h(r)\right] d s,
$$

Then, noticing that $Q_{g}$ is a linear operator, we have

$$
\widetilde{v}_{n}(\tau)=Q_{g} \widehat{v}_{n}(\tau)=Q_{g} v_{0}(\tau)+Q_{g} \xi(\tau)^{\top} Z_{n}+Q_{g} \eta(\tau)+o_{p}(1) .
$$


By construction, $Q_{g}(\xi(\tau))=0$, and by Khmaladze (1981), $Q_{g} v_{0}(\tau) \Rightarrow w_{0}(\tau)$, where $w_{0}$ is a standard Brownian motion. Thus

$$
\widetilde{v}_{n}(\tau) \Rightarrow w_{0}(\tau)+\widetilde{\eta}(\tau)
$$

Under the null hypothesis,

$$
\sup _{\tau \in \mathcal{T}}\left\|\tilde{v}_{n}(\tau)\right\| \Rightarrow \sup _{\tau \in \mathcal{T}}\left\|w_{0}(\tau)\right\|
$$

\section{Proof of Corollary 3}

We denote the transformation based on $\dot{g}_{n}$ as

$$
Q_{g_{n}}\left(\widehat{v}_{n}(\tau)\right)=\widehat{v}_{n}(\tau)-\int_{0}^{\tau}\left[\dot{g}_{n}(s)^{\top} C_{n}(s)^{-1} \int_{s}^{1} \dot{g}_{n}(r) d \widehat{v}_{n}(\tau)\right] d s .
$$

Noticing that

$$
\widehat{v}_{n}(\tau)=\sqrt{n} \varphi_{n}(\tau)\left[R_{n} \Omega_{n} R_{n}^{\top}\right]^{-1 / 2}\left[R_{n} \widehat{\beta}(\tau)-r_{n}-\Psi(\tau)\right]=v_{n}(\tau)+\xi_{n}(\tau)^{\top} Z_{n}+o_{p}(1)
$$

where $Z_{n}$ is an $O_{p}(1)$ quantity independent of $\tau$, by construction, $Q_{g_{n}}\left(g_{n}\right)=0$. Thus we have

$$
\begin{aligned}
& \widehat{v}_{n}(\tau)-\int_{0}^{\tau}\left[\dot{g}_{n}(s)^{\top} C_{n}(s)^{-1} \int_{s}^{1} \dot{g}_{n}(r) d \widehat{v}_{n}(r)\right] d s \\
= & v_{n}(\tau)-\int_{0}^{\tau}\left[\dot{g}_{n}(s)^{\top} C_{n}(s)^{-1} \int_{s}^{1} \dot{g}_{n}(r) d v_{n}(r)\right] d s+o_{p}(1) .
\end{aligned}
$$

Because $\dot{g}_{n}(r)$ is a consistent estimator of $\dot{g}(r)$ uniformly on $r \in \mathcal{T}=[\varepsilon, 1-\varepsilon]$, we have, for all $s \in \mathcal{T}$

$$
\left\|C(s)^{-1}\right\|=\left\|\left[\int_{s}^{1} \dot{g}(v) \dot{g}(v)^{\top} d v\right]^{-1}\right\| \leq\left\|\left[\int_{1-\varepsilon}^{1} \dot{g}(v) \dot{g}(v)^{\top} d v\right]^{-1}\right\|<\infty,
$$

and

$$
\begin{aligned}
\left\|C_{n}(s)^{-1}\right\| & =\left\|\left[\int_{s}^{1} \dot{g}_{n}(v) \dot{g}_{n}(v)^{\top} d v\right]^{-1}\right\| \\
& \leq\left\|\left[\int_{1-\varepsilon}^{1} \dot{g}_{n}(v) \dot{g}_{n}(v)^{\top} d v\right]^{-1}\right\| \\
& =\left\|\left[\int_{1-\varepsilon}^{1} \dot{g}(v) \dot{g}(v)^{\top} d v\right]^{-1}\right\|+o_{p}(1)<\infty .
\end{aligned}
$$

By assumption A.7, (A.1), and (A.2), we have

$$
\begin{aligned}
& \int_{0}^{\tau}\left[\dot{g}_{n}(s)^{\top} C_{n}(s)^{-1} \int_{s}^{1}\left[\dot{g}_{n}(r)-\dot{g}(r)\right] d v_{n}(r)\right] d s=o_{p}(1), \\
& \int_{0}^{\tau}\left[\left[\dot{g}_{n}(s)^{\top}-\dot{g}(s)^{\top}\right] C(s)^{-1} \int_{s}^{1} \dot{g}(r) d v_{n}(r)\right] d s=o_{p}(1) .
\end{aligned}
$$

Also notice that, under Assumption A.7, for all $s \in \mathcal{T}$,

$$
C(s)-C_{n}(s)=\int_{s}^{1}\left[\dot{g}(v) \dot{g}(v)^{\top}-\dot{g}_{n}(v) \dot{g}_{n}(v)^{\top}\right] d v=o_{p}(1),
$$


thus, by (A.3), (A.1), and (A.2),

$$
\begin{aligned}
& \int_{0}^{\tau}\left[\dot{g}_{n}(s)^{\top}\left[C_{n}(s)^{-1}-C(s)^{-1}\right] \int_{s}^{1} \dot{g}(r) d v_{n}(r)\right] d s \\
= & \int_{0}^{\tau}\left[\dot{g}_{n}(s)^{\top} C_{n}(s)^{-1}\left[C(s)-C_{n}(s)\right] C(s)^{-1} \int_{s}^{1} \dot{g}(r) d v_{n}(r)\right] d s \\
= & o_{p}(1) .
\end{aligned}
$$

Thus

$$
\begin{aligned}
& \int_{0}^{\tau}\left[\dot{g}_{n}(s)^{\top} C_{n}(s)^{-1} \int_{s}^{1} \dot{g}_{n}(r) d v_{n}(r)\right] d s-\int_{0}^{\tau}\left[\dot{g}(s)^{\top} C(s)^{-1} \int_{s}^{1} \dot{g}(r) d v_{n}(r)\right] d s \\
= & \int_{0}^{\tau}\left[\dot{g}_{n}(s)^{\top} C_{n}(s)^{-1} \int_{s}^{1}\left[\dot{g}_{n}(r)-\dot{g}(r)\right] d v_{n}(r)\right] d s \\
& +\int_{0}^{\tau}\left[\dot{g}_{n}(s)^{\top}\left[C_{n}(s)^{-1}-C(s)^{-1}\right] \int_{s}^{1} \dot{g}(r) d v_{n}(r)\right] d s \\
& +\int_{0}^{\tau}\left[\left[\dot{g}_{n}(s)^{\top}-\dot{g}(s)^{\top}\right] C(s)^{-1} \int_{s}^{1} \dot{g}(r) d v_{n}(r)\right] d s \\
= & o_{p}(1), \\
= & v_{n}(\tau)-\int_{0}^{\tau}\left[g(s)^{\top} C(s)^{-1} \int_{s}^{1} g(r) d v_{n}(r)\right] d s+o_{p}(1),
\end{aligned}
$$

and the result of Corollary 3 follows immediately.

\section{Appendix B. Asymptotic Critical Values}

Like many other Kolmogorov-Smirnov type tests (see, e.g. ?, the limiting distribution $\sup _{\tau \in \mathcal{T}}\left\|w_{0}(\tau)\right\|$ is dependent on the norm $\|\cdot\|$, the pre-specified $\mathcal{T}$ and the dimension parameter $q$. Notice that the transformation is generally unstable in the extreme right tails, and the uniform convergency of existing estimators of the density and score $\left(f\left(F^{-1}(s)\right)\right.$ and $\left.f^{\prime} / f\left(F^{-1}(s)\right)\right)$ usually requires that $\mathcal{T}$ be bounded away from zero and one, we consider a subset of $[0,1]$ whose closure lies in $(0,1)$.

We calculated the $1 \%, 5 \%$, and $10 \%$ critical values for the test statistic $\sup _{\tau \in \mathcal{T}}\left\|\widetilde{v}_{n}(\tau)\right\|$ based on simulations where the Brownian motion was approximated by a Gaussian random walk, using a sample size $n=2000$ and 20,000 replications. For the norm $\|\cdot\|$, we use the $\ell_{1}$ norm for a $q$ dimensional vector $x,\|x\|=\sum_{j=1}^{q}\left|x_{j}\right|$. Table 1 covers $\mathcal{T}=[\varepsilon, 1-\varepsilon]$ for $\varepsilon=0.05,0.1,0.15,0.2,0.25$, 0.3 , and $q=1,2, \ldots \ldots, 20$. Although conventionally we consider symmetric intervals $\mathcal{T}=[\varepsilon, 1-\varepsilon]$ for some small numbers $\varepsilon$, a much wider range of intervals $\mathcal{T}$ may be considered for the proposed tests. Critical values based other choices of the interval $\mathcal{T}$ and the dimension parameter $q$ can be similarly calculated. Gauss programs are available from the authors upon request.

Asymptotic Critical Values 


\begin{tabular}{|c|c|c|c|c|c|c|c|c|c|}
\hline & \multicolumn{3}{|c|}{$\delta=0.05$} & \multicolumn{3}{|c|}{$\delta=0.1$} & \multicolumn{3}{|c|}{$\delta=0.15$} \\
\hline & $1 \%$ & $5 \%$ & $10 \%$ & $1 \%$ & $5 \%$ & $10 \%$ & $1 \%$ & $5 \%$ & $10 \%$ \\
\hline$p=1$ & 2.721 & 2.140 & 1.872 & 2.640 & 2.102 & 1.833 & 2.573 & 2.048 & 1.772 \\
\hline$p=2$ & 4.119 & 3.393 & 3.011 & 4.034 & 3.287 & 2.946 & 3.908 & 3.199 & 2.866 \\
\hline$p=3$ & $\overline{5.350}$ & 4.523 & 4.091 & 5.267 & 4.384 & 3.984 & 5.074 & 4.269 & 3.871 \\
\hline$p=4$ & 6.548 & 5.560 & 5.104 & 6.340 & 5.430 & 4.971 & 6.148 & 5.284 & 4.838 \\
\hline$p=5$ & 7.644 & 6.642 & 6.089 & 7.421 & 6.465 & 5.931 & 7.247 & 6.264 & 5.758 \\
\hline$p=6$ & 8.736 & 7.624 & 7.047 & 8.559 & 7.412 & 6.852 & 8.355 & 7.197 & 6.673 \\
\hline$p=7$ & 9.876 & 8.578 & 7.950 & 9.573 & 8.368 & 7.770 & 9.335 & 8.125 & 7.536 \\
\hline$p=8$ & 10.79 & 9.552 & 8.890 & 10.53 & 9.287 & 8.662 & 10.35 & 9.044 & 8.412 \\
\hline$p=9$ & 11.81 & 10.53 & 9.820 & 11.55 & 10.26 & 9.571 & 11.22 & 9.963 & 9.303 \\
\hline$p=10$ & 12.91 & 11.46 & 10.72 & 12.54 & 11.17 & 10.43 & 12.19 & 10.85 & 10.14 \\
\hline$p=11$ & 14.03 & 12.41 & 11.59 & 13.58 & 12.10 & 11.29 & 13.27 & 11.77 & 10.98 \\
\hline$p=12$ & 15.00 & 13.34 & 12.52 & 14.65 & 13.00 & 12.20 & 14.26 & 12.61 & 11.86 \\
\hline$p=13$ & 15.93 & 14.32 & 13.37 & 15.59 & 13.90 & 13.03 & 15.22 & 13.48 & 12.69 \\
\hline$p=14$ & 16.92 & 15.14 & 14.28 & 16.52 & 14.73 & 13.89 & 16.12 & 14.34 & 13.48 \\
\hline$p=15$ & 17.93 & 16.11 & 15.19 & 17.53 & 15.67 & 14.76 & 17.01 & 15.24 & 14.36 \\
\hline$p=16$ & 18.85 & 16.98 & 16.06 & 18.46 & 16.56 & 15.65 & 17.88 & 16.06 & 15.22 \\
\hline$p=17$ & 19.68 & 17.90 & 16.97 & 19.24 & 17.44 & 16.53 & 18.78 & 16.93 & 16.02 \\
\hline$p=18$ & 20.63 & 18.83 & 17.84 & 20.21 & 18.32 & 17.38 & 19.70 & 17.80 & 16.86 \\
\hline$p=19$ & 21.59 & 19.72 & 18.73 & 21.06 & 19.24 & 18.24 & 20.53 & 18.68 & 17.70 \\
\hline \multirow[t]{3}{*}{$p=20$} & 22.54 & 20.58 & 19.62 & 22.02 & 20.11 & 19.11 & 21.42 & 19.52 & 18.52 \\
\hline & & $\delta=0.2$ & & & $\delta=0.25$ & & & $\delta=0.3$ & \\
\hline & $1 \%$ & $5 \%$ & $10 \%$ & $1 \%$ & $5 \%$ & $10 \%$ & $1 \%$ & $5 \%$ & $10 \%$ \\
\hline$p=1$ & 2.483 & 1.986 & 1.730 & 2.420 & 1.923 & 1.664 & 2.320 & 1.849 & 1.602 \\
\hline$p=2$ & 3.742 & 3.100 & 2.781 & 3.633 & 3.000 & 2.693 & 3.529 & 2.904 & 2.602 \\
\hline$p=3$ & 4.893 & 4.133 & 3.749 & 4.737 & 4.018 & 3.632 & 4.599 & 3.883 & 3.529 \\
\hline$p=4$ & 6.023 & 5.091 & 4.684 & 5.818 & 4.948 & 4.525 & 5.599 & 4.807 & 4.365 \\
\hline$p=5$ & 6.985 & 6.070 & 5.594 & 6.791 & 5.853 & 5.406 & 6.577 & 5.654 & 5.217 \\
\hline$p=6$ & 8.147 & 6.985 & 6.464 & 7.922 & 6.760 & 6.241 & 7.579 & 6.539 & 6.024 \\
\hline$p=7$ & 9.094 & 7.887 & 7.299 & 8.856 & 7.611 & 7.064 & 8.542 & 7.357 & 6.832 \\
\hline$p=8$ & 10.03 & 8.775 & 8.169 & 9.685 & 8.510 & 7.894 & 9.413 & 8.211 & 7.633 \\
\hline$p=9$ & 10.90 & 9.672 & 9.018 & 10.61 & 9.346 & 8.737 & 10.27 & 9.007 & 8.400 \\
\hline$p=10$ & 11.89 & 10.52 & 9.843 & 11.48 & 10.17 & 9.517 & 11.15 & 9.832 & 9.192 \\
\hline$p=11$ & 12.85 & 11.35 & 10.66 & 12.48 & 10.99 & 10.28 & 12.06 & 10.62 & 9.929 \\
\hline$p=12$ & 13.95 & 12.22 & 11.48 & 13.54 & 11.82 & 11.11 & 12.96 & 11.43 & 10.74 \\
\hline$p=13$ & 14.86 & 13.09 & 12.31 & 14.34 & 12.66 & 11.93 & 13.82 & 12.24 & 11.51 \\
\hline$p=14$ & 15.69 & 13.92 & 13.11 & 15.26 & 13.46 & 12.67 & 14.64 & 13.03 & 12.28 \\
\hline$p=15$ & 16.55 & 14.77 & 13.91 & 16.00 & 14.33 & 13.47 & 15.46 & 13.85 & 13.05 \\
\hline$p=16$ & 17.41 & 15.58 & 14.74 & 16.81 & 15.09 & 14.26 & 16.25 & 14.61 & 13.78 \\
\hline$p=17$ & 18.19 & 16.43 & 15.58 & 17.59 & 15.95 & 15.06 & 17.04 & 15.39 & 14.54 \\
\hline$p=18$ & 19.05 & 17.30 & 16.37 & 18.49 & 16.78 & 15.83 & 17.85 & 16.14 & 15.30 \\
\hline$p=19$ & 19.96 & 18.09 & 17.17 & 19.40 & 17.50 & 16.64 & 18.78 & 16.94 & 16.05 \\
\hline$p=20$ & 20.81 & 18.95 & 17.97 & 20.14 & 18.30 & 17.38 & 19.48 & 17.74 & 16.79 \\
\hline
\end{tabular}




\section{REFERENCES}

Andrews, D. W. K. (1993): “Tests for Parameter Instability and Structural Change With Unknown Change Point," Econometrica, 61, 821-856.

BAI, J. (1998): “Testing parametric conditional distributions of dynamic models," preprint.

Bassett, Gilbert, J., And R. Koenker (1982): “An Empirical Quantile Function for Linear Models With Tid Errors," J. of Am. Stat. Assoc., 77, 407-415.

Bickel, P. J. (1982): “On Adaptive Estimation," AnlsStat, 10, 647-671.

Bilias, Y., S. Chen, AND Z. Ying (1999): "Simple resampling methods for censored regression quantiles," preprint.

Bofinger, E. (1975): "Estimation of a density function using order statistics," Australian J. of Statistics, 17, 1-7.

Corson, W., P. Decker, S. Dunstan, and S. Keransky (1992): "Pennsylvania 'reemployment bonus' demonstration: Final Report," Unemployment Insurance Occasional Paper, 92.

Cox, D. D. (1985): “A Penalty Method for Nonparametric Estimation of the Logarithmic Derivative of a Density Function," AnInStMa, 37, 271-288.

Darling, D. (1955): “The Cramér-Smirnov test in the parametric case," Anls Stat, 26, 1-20.

Doksum, K. (1974): "Empirical Probability Plots and Statistical Inference for Nonlinear Models in the Two-sample Case," Annals of Stat., 2, 267-277.

Doов, J. (1949): "A Heuristic Approach to the Kolmogorov-Smirnov theorems," Annals of Stat., $20,393-403$.

Durbin, J. (1973): Distribution theory for tests based on the sample distribution function. SIAM. Fleming, T., And D. Harrington (1991): Counting Processes and Survival Processes. Wiley. Gutenbrunner, C., AND J. JuRečKová (1992): "Regression quantile and regression rank score process in the linear model and derived statistics," Ann. Statist., 20, 305-330.

Gutenbrunner, C., J. Jurečková , R. Koenker, And S. Portnoy (1993): “Tests of linear hypotheses based on regression rank scores," J. of Nonparametric Statistics, 2, 307-33.

HAHN, J. (1995): "Bootstrapping quantile regression models," Econometric Theory, 11, $105-121$.

HE, X., AND F. Hu (1999): "Markov chain marginal bootstrap," preprint.

Horowitz, J. (1998): "Bootstrap methods for median regression models," Econometrica, 66, 13271352.

Hsieh, D. A., And C. F. Manski (1987): "Monte Carlo Evidence on Adaptive Maximum Likelihood Estimation of a Regression," AnlsStat, 15, 541-551.

Khmaladze, E. V. (1981): "Martingale Approach in the Theory of Goodness-of-fit Tests," Theory of Prob. and its Apps, 26, 240-257.

Kiefer, J. (1959): "K-sample analogues of the Kolmogorov-Smirnov and Cramér-von Mises tests," Ann. Math. Statist, 30, 420-447.

Koenker, R., And G. Bassett (1982): “Tests of linear hypotheses and $l_{1}$ estimation," Econometrica, 50, 1577-1584.

Koenker, R., AND Y. Bilias (1999): "Quantile Regression for Duration Data: A Reappraisal of the Pennsylvania Reemployment Bonus Experiments," forthcoming.

Koenker, R., AND J. Machado (1999): "Goodness of fit and related inference processes for quantile regression," J. of Am. Stat. Assoc., pp. 11296-1310.

Koenker, R., And S. Portnoy (1987): "L-estimation for Linear Models," J. of Am. Stat. Assoc., $82,851-857$.

Koul, H., And W. Stute (1999): “Nonparametric Model Checks for Time Series," Annals of Stat., 27, 204-236.

Lehmann, E. (1974): Nonparametrics: Statistical Methods based on Ranks. Holden-Day, San Francisco. 
Meyer, B. (1995): "Lessons from the U.S. unemployment insurance experiments," Journal of Economic Literature, 33, 191-131.

(1996): "What have we learned from the Illinois reemployment bonus experiment," Journal of Labor Economics, 14, 26-51.

NG, P. T. (1994): "Smoothing Spline Score Estimation," SIA MSSC, 15, 1003-1025.

Portnoy, S., And R. Koenker (1989): “Adaptive L-estimation of linear models," Ann. Statist, $17,362-381$.

Serfling, R. (1980): Approximation Theorems of Mathematical Statistics. New York: Wiley.

Sheather, S., AND J. MARITZ (1983): "An estimate of the asymptotic standard error of the sample medi an," Australian J. of Statistics, 25, 109-122.

Shorack, G., And J. Wellner (1986): Empirical Processes with Applications to Statistics. Wiley. Siddiqui, M. (1960): "Distribution of Quantiles from a Bivariate Population," Journal of Research of the National Bureau of Standards, 64B, 145-150.

Stigler, S. (1980): "Stigler's Law of Eponymy," Transactions of the New York Academy of Sciences, $39,147-157$.

Stute, W. (1997): “Nonparametric Model Checks for Regression,” AnlsStat, 25, 613-641.

Welsh, A. H. (1988): "Asymptotically Efficient Estimation of the Sparsity Function At a Point," Stat. and Prob. Letters, 6, 427-432. 